\title{
Higher-Order Rogue Wave and Rational Soliton Solutions of Discrete Complex mKdV Equations
}

\author{
Xiao-Yong Wen* \\ School of Applied Science, Beijing Information Science and Technology University, \\ Beijing 100192, China.
}

Received 2 August 2017; Accepted (in revised version) 10 October 2017.

\begin{abstract}
The generalised perturbation $(n, N-n)$-fold Darboux transformation is used to derive new higher-order rogue wave and rational soliton solutions of the discrete complex mKdV equations. The structure of such waves and details of their evolution are investigated via numerical simulations, showing that the strong interaction yields weak oscillation and stability whereas the weak interaction is associated with strong oscillation and instability. A small noise has a weak influence on the wave propagation for the strong interaction, but substantially changes the wave behaviour in the weak interaction case.
\end{abstract}

AMS subject classifications: 35Q51, 35Q53, 37K05, 37K10

Key words: Discrete complex mKdV equation, modulational instability, generalised perturbation $(n, N-n)$-fold Darboux transformation, higher-order rogue wave solutions, higher-order rational soliton solutions.

\section{Introduction}

Nonlinear partial differential equations (NPDEs) frequently arise in scientific fields such as fluid dynamics, plasma physics or optics [31-36,39], and their discrete analogues in areas such as population dynamics, plasma physics, real ionic fluids, and the evolution of slowly varying envelopes of electric fields $[1,2,4,5,16-18,20-23,27,29,30]$. Physicists and mathematicians have found rogue wave solutions of NPDEs in nonlinear optics, plasma physics, Bose-Einstein condensates, and financial markets [10, 11, 13, 19, 47, 48]. For continuous NPDEs, the rogue wave solutions have been constructed using the Darboux transformation and its generalisations $[10,11,13,15]$. Discrete rogue wave solutions of the single-component Ablowitz-Ladik and discrete Hirota equations [6-9], the focusing and defocusing Ablowitz-Ladik equations [26] and a discrete nonlocal nonlinear Schrödinger equation [46] have also been considered. Nevertheless, the discrete rogue wave solutions of discrete integrable NPDEs remain little studied.

*Corresponding author. Email address: xiaoyongwen@163.com (X.-Y. Wen) 
Let us consider the discrete complex mKdV equation

$$
u_{n, t}=\left(1+\left|u_{n}\right|^{2}\right)\left(u_{n+1}-u_{n-1}\right),
$$

where $u_{n}=u_{n}(t)=u(n, t)$ is the complex field of discrete and time variables $n$ and $t$ respectively, $u_{n, t}$ denotes the derivative $d u_{n} / d t$, and $\left|u_{n}\right|^{2}:=u_{n} u_{n}^{*}$ where the asterisk denotes complex conjugation. Eq. (1.1) has been discussed in Ref. [8], and may be viewed as a discrete version of the continuous complex mKdV equation

$$
i u_{t}+\frac{1}{2} u_{x x x}+3|u|^{2} u_{x}=0
$$

with many applications - e.g. in electrodynamics, electromagnetics, density stratification, elastic media and traffic flow $[25,49,50]$.

Let $E: f(n, t) \rightarrow f(n+1, t)$ and $E^{-1}: f(n, t) \rightarrow f(n-1, t), n \in \mathbb{Z}, t \in \mathbb{R}$ be the shift operators, where sometimes the variable $t$ is repressed so $f(n, t)$ is abbreviated as $f(n)$. According to Refs. $[3,40]$, the Lax pairs associated with Eq. (1.1) are

$$
\begin{aligned}
& E \varphi_{n}=U_{n} \varphi_{n}=\left(\begin{array}{cc}
\lambda^{2} & \lambda u_{n} \\
-\lambda u_{n}^{*} & 1
\end{array}\right) \varphi_{n}, \\
& \varphi_{n, t}=V_{n} \varphi_{n}=\left(\begin{array}{cc}
\frac{\lambda^{2}}{2}-\frac{1}{2 \lambda^{2}}+u_{n} u_{n-1}^{*} & \lambda u_{n}+\frac{u_{n-1}}{\lambda} \\
-\lambda u_{n-1}^{*}-\frac{u_{n}^{*}}{\lambda} & -\frac{\lambda^{2}}{2}+\frac{1}{2 \lambda^{2}}+u_{n}^{*} u_{n-1}
\end{array}\right) \varphi_{n},
\end{aligned}
$$

where the eigenvalue parameter $\lambda$ does not depend on $n$ and $t, \varphi_{n}:=\left(\varphi_{1, n}, \varphi_{2, n}\right)^{T}$ is the vector eigenfunction and $T$ denotes the transposition. It is easily seen that the compatibility condition $\varphi_{n, t}=\varphi_{t, n}$ for Eqs. (1.2) and (1.3) yields Eq. (1.1). The approach adopted in Ref. [8] to obtain the two lowest-order rogue wave solutions of the discrete Hirota equation can also be used to produce low-order rogue wave solutions of Eq. (1.1).

In Section 2, a discrete integrable hierarchy associated with Eq. (1.1) is presented and new discrete integrable NPDEs are obtained. Section 3 deals with the modulation instability of the general plane-wave states of Eq. (1.1). Section 4 is devoted to a discrete version of the generalised perturbation ( $n, N-n)$-fold Darboux transformation of Eq. (1.1), used to study integrable continuous NPDEs $[41,42,44,45]$ and the discrete coupled AblowitzLadik equation [43]. These ideas are applied to Eq. (1.1) in Section 5, and a higher-order discrete rogue wave solution that differs from the rogue wave solutions of discrete coupled Ablowitz-Ladik equations in Ref. [43] is obtained. Moreover, we also derive higher-order discrete rational soliton solutions. To the best of the author's knowledge, such solutions for discrete NPDEs have not been reported in literature before. Concluding remarks are in Section 6 .

\section{Discrete Integrable Hierarchy}

Let us now consider the discrete matrix spectral problem

$$
E \varphi_{n}=U_{n}(u, \lambda) \varphi_{n}, U_{n}(u, \lambda)=\left(\begin{array}{cc}
\lambda^{2} & \lambda u_{n} \\
\lambda v_{n} & \beta
\end{array}\right),
$$


where $\lambda$ is a spectral parameter such that $\lambda_{t}=0, \beta$ is an arbitrary constant, $\varphi_{n}=\left(\varphi_{1, n}, \varphi_{2, n}\right)^{T}$ is an eigenfunction vector, and $u=\left(u_{n}, v_{n}\right)^{T}$ is the potential function. To obtain the hierarchy of Eq. (1.1) associated with the spectral problem (2.1), it is convenient to use the Tu scheme $[14,24,28]$. Let

$$
\Gamma_{1}=\left(\begin{array}{cc}
A_{n} & B_{n} \\
C_{n} & -A_{n}
\end{array}\right)
$$

The discrete stationary zero-curvature equation

$$
\left(E \Gamma_{1}\right) U_{n}-U_{n} \Gamma_{1}=0
$$

is equivalent to the system of equations

$$
\begin{aligned}
& \lambda^{2}\left(A_{n+1}-A_{n}\right)+\lambda v_{n} B_{n+1}-\lambda u_{n} C_{n}=0, \\
& \lambda u_{n}\left(A_{n+1}+A_{n}\right)-\lambda^{2} B_{n}+\beta B_{n+1}=0, \\
& \lambda^{2} C_{n+1}-\beta C_{n}-\lambda v_{n}\left(A_{n+1}+A_{n}\right)=0, \\
& \lambda u_{n} C_{n+1}-A_{n+1}+A_{n}-\lambda v_{n} B_{n}=0 .
\end{aligned}
$$

Substituting $A_{n}=\sum_{j=0}^{\infty} A_{n}^{(j)} \lambda^{-2 j}, B_{n}=\sum_{j=0}^{\infty} B_{n}^{(j)} \lambda^{-2 j+1}, C_{n}=\sum_{j=0}^{\infty} C_{n}^{(j)} \lambda^{-2 j+1}$ into Eqs. (2.2) leads to the initial conditions $B_{n}^{(0)}=0, C_{n+1}^{(0)}=0$ and the recursion relations

$$
\begin{aligned}
& A_{n+1}^{(j)}-A_{n}^{(j)}+v_{n} B_{n+1}^{(j)}-u_{n} C_{n}^{(j)}=0, \quad j \geq 0, \\
& u_{n}\left(A_{n+1}^{(j)}+A_{n}^{(j)}\right)-B_{n}^{(j+1)}-\beta B_{n+1}^{(j)}=0, \quad j \geq 0, \\
& C_{n+1}^{(j+1)}-\beta C_{n}^{(j)}-v_{n}\left(A_{n+1}^{(j)}+A_{n}^{(j)}\right)=0, \quad j \geq 0, \\
& u_{n} C_{n+1}^{(j+1)}-v_{n} B_{n}^{(j+1)}-\beta\left(A_{n+1}^{(j)}-A_{n}^{(j)}\right)=0, \quad j \geq 0 .
\end{aligned}
$$

Setting $A_{n}^{(0)}=-1 / 2$, the recursion relations (2.3) uniquely determine $A_{n}^{(j)}, B_{n}^{(j)}, C_{n}^{(j)}(j \geq 1)$. For example,

$$
\begin{aligned}
B_{n}^{(1)}= & -u_{n}, \quad C_{n+1}^{(1)}=-v_{n}, \quad A_{n}^{(1)}=u_{n} v_{n-1}, \\
B_{n}^{(2)}= & u_{n}\left(u_{n+1} v_{n}+u_{n} v_{n-1}\right)-\beta u_{n+1}, \quad C_{n+1}^{(2)}=v_{n}\left(u_{n+1} v_{n}+u_{n} v_{n-1}\right)-\beta v_{n-1}, \\
A_{n}^{(2)}= & -u_{n}^{2} v_{n-1}^{2}-u_{n} v_{n-1}\left(u_{n+1} v_{n}+u_{n-1} v_{n-2}\right)+\beta\left(u_{n+1} v_{n-1}+u_{n} v_{n-2}\right), \\
B_{n}^{(3)}= & \beta\left(u_{n}\left(u_{n+2} v_{n+1}+u_{n+1} v_{n}\right)-\beta u_{n+2}\right)+u_{n}\left(-u_{n+1}^{2} v_{n}^{2}-u_{n+1} v_{n}\left(u_{n} v_{n+1}+u_{n} v_{n-1}\right)\right. \\
& \left.+\beta\left(u_{n+2} v_{n}+u_{n+1} v_{n-1}\right)-u_{n}^{2} v_{n-1}^{2}-u_{n} v_{n-1}\left(u_{n+1} v_{n}+u_{n-1} v_{n-2}\right)+\beta\left(u_{n+1} v_{n-1}+u_{n} v_{n-2}\right)\right), \\
C_{n+1}^{(3)}= & \beta\left(v_{n-1}\left(u_{n} v_{n-1}+u_{n-1} v_{n-2}\right)-\beta v_{n-2}\right)+v_{n}\left(-u_{n+1}^{2} v_{n}^{2}-u_{n+1} v_{n}\left(u_{n} v_{n+1}+u_{n} v_{n-1}\right)\right. \\
& \left.+\beta\left(u_{n+2} v_{n}+u_{n+1} v_{n-1}\right)-u_{n}^{2} v_{n-1}^{2}-u_{n} v_{n-1}\left(u_{n+1} v_{n}+u_{n-1} v_{n-2}\right)+\beta\left(u_{n+1} v_{n-1}+u_{n} v_{n-2}\right)\right) .
\end{aligned}
$$

If $\Gamma_{1}^{(m)}$ is defined by

$$
\Gamma_{1}^{(m)}:=\lambda^{2 m} \Gamma_{1}=\left(\begin{array}{cc}
\sum_{j=0}^{m} A_{n}^{(j)} \lambda^{2 m-2 j} & \sum_{j=0}^{m} B_{n}^{(j)} \lambda^{2 m-2 j+1} \\
\sum_{j=0}^{m} C_{n}^{(j)} \lambda^{2 m-2 j+1} & -\sum_{j=0}^{m} A_{n}^{(j)} \lambda^{2 m-2 j}
\end{array}\right), \quad m \geq 0,
$$


then the relations (2.3) imply

$$
\left(E \Gamma_{1}^{(m)}\right) U_{n}-U_{n} \Gamma_{1}^{(m)}=\left(\begin{array}{cc}
0 & \lambda B_{n}^{(m+1)} \\
-\lambda C_{n+1}^{(m+1)} & -\beta\left(A_{n+1}^{(m)}-A_{n}^{(m)}\right)
\end{array}\right) .
$$

Let us introduce the matrix

$$
\Delta_{n}^{(m)}=\left(\begin{array}{cc}
0 & 0 \\
0 & A_{n}^{(m)}
\end{array}\right)
$$

and set $V_{n}^{(m)}:=\Gamma_{1}^{(m)}+\Delta_{n}^{(m)}, m \geq 0$ such that

$$
\left(E V_{n}^{(m)}\right) U_{n}-U_{n} V_{n}^{(m)}=\left(\begin{array}{cc}
0 & \lambda B_{n+1}^{(m)}-\lambda u_{n} A_{n}^{(m)} \\
\lambda v_{n} A_{n+1}^{(m)}-\lambda C_{n+1}^{(m+1)} & 0
\end{array}\right) .
$$

Let us also assume that the eigenfunctions $\varphi_{n}$ of Eq. (2.1) satisfy the equations

$$
\varphi_{n, t_{m}}=V_{n}^{(m)} \varphi_{n}, \quad m \geq 0 .
$$

The compatibility conditions of Eqs. (2.1) and (2.4) have the form $E \varphi_{n, t_{m}}=E\left(\varphi_{n}\right)_{t_{m}}$ and are equivalent to

$$
U_{n, t_{m}}=\left(E V_{n}^{(m)}\right) U_{n}-U_{n} V_{n}^{(m)}, \quad m \geq 0,
$$

which yields the positive discrete integrable hierarchy

$$
\begin{aligned}
& u_{n, t_{m}}=B_{n+1}^{(m)}-u_{n} A_{n}^{(m)}, \\
& v_{n, t_{m}}=v_{n} A_{n+1}^{(m)}-C_{n+1}^{(m+1)} .
\end{aligned}
$$

In order to derive the negative discrete integrable hierarchy associated with Eq. (2.1), let us consider the spectral problems

$$
\varphi_{n, t_{m}}=\Gamma_{2}^{(m)} \varphi_{n}, \quad m \geq 0,
$$

where

$$
\Gamma_{2}^{(m)}=\left(\begin{array}{cc}
\sum_{j=0}^{m} a_{n}^{(j)} \lambda^{-2 m+2 j} & \sum_{j=0}^{m} b_{n}^{(j)} \lambda^{-2 m+2 j-1} \\
\sum_{j=0}^{m} c_{n}^{(j)} \lambda^{-2 m+2 j-1} & -\sum_{j=0}^{m} a_{n}^{(j)} \lambda^{-2 m+2 j}
\end{array}\right)+\left(\begin{array}{cc}
a_{n}^{(m)} & 0 \\
0 & 0
\end{array}\right)
$$

The discrete zero-curvature equations $U_{n, t_{m}}=\left(E \Gamma_{2}^{(m)}\right) U_{n}-U_{n} \Gamma_{2}^{(m)}$ give the hierarchy

$$
\begin{aligned}
& u_{n, t_{m}}=b_{n+1}^{(m+1)}-u_{n} a_{n+1}^{(m)}, \\
& v_{n, t_{m}}=c_{n}^{(m+1)}+v_{n} a_{n}^{(m)},
\end{aligned}
$$


with the corresponding first recursive relations

$$
\begin{aligned}
& a_{n}^{(0)}=-1 / 2, \quad b_{n+1}^{(0)}=c_{n}^{(0)}=0, \quad b_{n+1}^{(1)}=u_{n} / \beta, \quad c_{n}^{(1)}=v_{n} / \beta, \quad a_{n}^{(1)}=v_{n} u_{n-1} / \beta, \\
& a_{n}^{(2)}=-\left(v_{n}^{2} u_{n-1}+u_{n-1} u_{n-2} v_{n} v_{n-1}+u_{n} u_{n-1} v_{n} v_{n+1}-\beta\left(u_{n-1} v_{n+1}+u_{n-2} v_{n}\right)\right) / \beta^{4}, \\
& b_{n+1}^{(2)}=-\left(u_{n}^{2} v_{n+1}+u_{n} u_{n-1} v_{n}-\beta u_{n-1}\right) / \beta^{3}, \quad c_{n}^{(2)}=-\left(v_{n}^{2} u_{n-1}+u_{n} v_{n+1} v_{n}-\beta v_{n+1}\right) / \beta^{3}, \\
& b_{n+1}^{(3)}=\left(b_{n}^{(2)}-u_{n}\left(a_{n+1}^{(2)}+a_{n}^{(2)}\right)\right) / \beta, \quad c_{n}^{(3)}=\left(c_{n+1}^{(2)}-v_{n}\left(a_{n+1}^{(2)}+a_{n}^{(2)}\right)\right) / \beta .
\end{aligned}
$$

A more general discrete integrable hierarchy associated with Eq. (2.1) can be obtained as follows. Let $\mu_{1}$ and $\mu_{2}$ be constants and let $P_{n}^{(m)}:=\mu_{1} V_{n}^{(m)}+\mu_{2} \Gamma_{2}^{(m)}$. Let us consider the spectral problem

$$
\varphi_{n, t_{m}}=P_{n}^{(m)} \varphi_{n}, \quad m \geq 0 .
$$

The discrete zero-curvature equations $U_{n, t_{m}}=\left(E P_{2}^{(m)}\right) U_{n}-U_{n} P_{2}^{(m)}$ produce the hierarchy

$$
\begin{aligned}
& u_{n, t_{m}}=\mu_{1}\left(B_{n+1}^{(m)}-u_{n} A_{n}^{(m)}\right)+\mu_{2}\left(b_{n+1}^{(m+1)}-u_{n} a_{n+1}^{(m)}\right), \\
& v_{n, t_{m}}=\mu_{1}\left(v_{n} A_{n+1}^{(m)}-C_{n+1}^{(m+1)}\right)+\mu_{2}\left(c_{n}^{(m+1)}+v_{n} a_{n}^{(m)}\right) .
\end{aligned}
$$

If $\mu_{1}=0, \mu_{2}=1$, then (2.7) becomes the system (2.5) and if $\mu_{1}=1, \mu_{2}=0$ one has the system (2.6). On letting $\mu_{1}=1$ and $\mu_{2}=-1$, if $m=1$ the first nontrivial result is then

$$
\begin{aligned}
& u_{n, t_{1}}=-u_{n+1}\left(u_{n} v_{n}-1\right)+u_{n-1}\left(u_{n} v_{n}-1\right) / \beta^{2}, \\
& v_{n, t_{1}}=v_{n-1}\left(u_{n} v_{n}-1\right)-v_{n+1}\left(u_{n} v_{n}-1\right) / \beta^{2},
\end{aligned}
$$

and the time part of the Lax pairs for (2.8) is

$$
V_{n}^{(1)}=\left(\begin{array}{cc}
\lambda^{2} / 2-1 /\left(2 \lambda^{2}\right)-u_{n} v_{n-1} & \lambda u_{n}+u_{n-1} /(\beta \lambda) \\
\lambda v_{n-1}+v_{n} /(\beta \lambda) & -\lambda^{2} / 2+1 /\left(2 \lambda^{2}\right)-v_{n} u_{n-1} / \beta^{2}
\end{array}\right) .
$$

If $\beta=1$ and $v_{n}, u_{n}$ satisfy the symmetry reduction condition $v_{n}=\sigma u_{n}$, then the equations of the system (2.8) are compatible such that the discrete mKdV equation with $\sigma=-1$ is (cf. the Volterra lattice equation in Ref. [40])

$$
u_{n, t_{1}}=\left(1-\sigma u_{n}^{2}\right)\left(u_{n+1}-u_{n-1}\right) .
$$

On the other hand, choosing $\beta=1$ and $\sigma=-1$ yields the discrete complex mKdV equation

$$
u_{n, t_{1}}=\left(1-\sigma\left|u_{n}\right|^{2}\right)\left(u_{n+1}-u_{n-1}\right),
$$

which is actually Eq. (1.1) with the Lax pairs (1.2) and (1.3).

Changing the symmetry reduction conditions produces other equations. For example, taking $\beta=1$ and $v_{n}=\sigma u(-n, t)$ yields a new nonlocal discrete mKdV equation - viz.

$$
u_{n, t_{1}}=\left(1-\sigma u_{n} u(-n, t)\right)\left(u_{n+1}-u_{n-1}\right)
$$


Further, if $\beta=1$ and $v_{n}=\sigma u^{*}(-n, t)$ one obtains a new nonlocal discrete complex mKdV equation

$$
u_{n, t_{1}}=\left(1-\sigma u_{n} u^{*}(-n, t)\right)\left(u_{n+1}-u_{n-1}\right) .
$$

For $m=2$ and $\beta=1$, the second equations in this hierarchy have the form

$$
\begin{aligned}
u_{n, t_{2}}= & \left(1-u_{n} v_{n}\right)\left[v_{n}\left(u_{n-1}^{2}-u_{n+1}^{2}\right)+u_{n}\left(u_{n-1} v_{n+1}-v_{n-1} u_{n+1}\right)\right. \\
& \left.+u_{n+2}\left(1-u_{n+1} v_{n+1}\right)-u_{n-2}\left(1-u_{n-1} v_{n-1}\right)\right], \\
v_{n, t_{2}}= & \left(1-u_{n} v_{n}\right)\left[u_{n}\left(v_{n-1}^{2}-v_{n+1}^{2}\right)+v_{n}\left(v_{n-1} u_{n+1}-u_{n-1} v_{n+1}\right)\right. \\
& \left.+v_{n+2}\left(1-u_{n+1} v_{n+1}\right)-v_{n-2}\left(1-u_{n-1} v_{n-1}\right)\right],
\end{aligned}
$$

with the corresponding time part of the associated Lax pairs

$$
V_{n}^{(2)}=\left(\begin{array}{ll}
V_{11} & V_{12} \\
V_{21} & V_{22}
\end{array}\right)
$$

where

$$
\begin{aligned}
V_{11}= & (1 / 2) \lambda^{4}-u_{n} v_{n-1} \lambda^{2}-u_{n+1} v_{n-1}\left(1-u_{n} v_{n}\right)-u_{n} v_{n-2}\left(1-u_{n-1} v_{n-1}\right) \\
& +u_{n}^{2} v_{n-1}^{2}-1 /\left(2 \lambda^{4}\right)+u_{n-1} v_{n} / \lambda^{2}, \\
V_{12}= & -\left(u_{n} v_{n} u_{n+1}+u_{n}^{2} v_{n-1}-u_{n+1}\right) \lambda+\lambda^{3} u_{n}+u_{n-1} / \lambda^{3} \\
& +\left(u_{n-2}-u_{n-1}^{2} v_{n}-u_{n-1} u_{n-2} v_{n-1}\right) / \lambda, \\
V_{21}= & -\left(-v_{n-2}+v_{n-1}^{2} u_{n}+v_{n-1} v_{n-2} u_{n-1}\right) \lambda+\lambda^{3} v_{n-1}+v_{n} / \lambda^{3} \\
& +\left(v_{n+1}-u_{n} v_{n} v_{n+1}-v_{n}^{2} u_{n-1}\right) / \lambda, \\
V_{22}= & -(1 / 2) \lambda^{4}+u_{n} v_{n-1} \lambda^{2}+1 /\left(2 \lambda^{4}\right)-u_{n-1} v_{n} / \lambda^{2}-u_{n-1} v_{n+1}\left(1-u_{n} v_{n}\right) \\
& -u_{n-2} v_{n}\left(1-u_{n-1} v_{n-1}\right)+u_{n-1}^{2} v_{n}^{2} .
\end{aligned}
$$

For the symmetry reduction condition $v_{n}=\sigma u_{n}$, system (2.10) yields a new higher-order discrete mKdV equation with $\sigma=-1$ :

$$
u_{n, t_{2}}=\left(1-\sigma u_{n}^{2}\right)\left[\sigma u_{n}\left(u_{n-1}^{2}-u_{n+1}^{2}\right)+u_{n+2}\left(1-\sigma u_{n+1}^{2}\right)-u_{n-2}\left(1-\sigma u_{n-1}^{2}\right)\right],
$$

which will be called the higher-order Volterra lattice equation.

For the symmetry reduction condition $v_{n}=\sigma u_{n}^{*}$, the system (2.10) produces another new higher-order discrete complex mKdV equation with $\sigma=-1-$ viz.

$$
\begin{aligned}
u_{n, t_{2}}= & \left(1-\sigma\left|u_{n}\right|^{2}\right)\left[\sigma u_{n}^{*}\left(u_{n-1}^{2}-u_{n+1}^{2}\right)+\sigma u_{n}\left(u_{n-1} u_{n+1}^{*}-u_{n-1}^{*} u_{n+1}\right)\right. \\
& \left.+u_{n+2}\left(1-\sigma\left|u_{n+1}\right|^{2}\right)-u_{n-2}\left(1-\sigma\left|u_{n-1}\right|^{2}\right)\right] .
\end{aligned}
$$

The symmetry reduction condition $v_{n}=\sigma u(-n, t)$ with $\beta=1$ then yields a new higherorder reverse discrete nonlocal complex $\mathrm{mKdV}$ equation

$$
\begin{aligned}
u_{n, t_{2}}= & \left(1-\sigma u_{n} u(-n, t)\right)\left[\sigma u(-n, t)\left(u_{n-1}^{2}-u_{n+1}^{2}\right)+\sigma u_{n}\left(u_{n-1} u(-n-1, t)-u_{n+1} u(-n+1, t)\right)\right. \\
& \left.+u_{n+2}\left(1-\sigma u_{n+1} u(-n-1, t)\right)-u_{n-2}\left(1-\sigma u_{n-1} u(-n+1, t)\right)\right] .
\end{aligned}
$$


Further, the conditions $\beta=1$ and $v_{n}=\sigma u^{*}(-n, t)$ lead to new complex higher-order reverse discrete nonlocal discrete complex mKdV equation

$$
\begin{aligned}
u_{n, t_{2}}= & \left(1-\sigma u_{n} u^{*}(-n, t)\right)\left[\sigma u^{*}(-n, t)\left(u_{n-1}^{2}-u_{n+1}^{2}\right)\right. \\
& +\sigma u_{n}\left(u_{n-1} u^{*}(-n-1, t)-u_{n+1} u^{*}(-n+1, t)\right) \\
& \left.+u_{n+2}\left(1-\sigma u_{n+1} u^{*}(-n-1, t)\right)-u_{n-2}\left(1-\sigma u_{n-1} u^{*}(-n+1, t)\right)\right] .
\end{aligned}
$$

\section{Modulational Instability of the Plane-Wave States}

Let $c e^{\left.i\left[a x+2 \sin (a)\left(1+c^{2}\right) t\right]\right]}$ be a plane wave with a real amplitude $c$ and real wave number a. For the perturbation

$$
u_{n}=\left[c+\varepsilon Q_{n}\right] e^{\left.i\left[a x+2 \sin (a)\left(1+c^{2}\right) t\right]\right)},
$$

substitution into Eq. (1.1) yields the linearised equation

$$
\begin{aligned}
& Q_{n, t}+2 i \sin (a) Q_{n}-Q_{n+1} c^{2} \cos (a)-2 i c^{2} Q_{n}^{*} \sin (a)-Q_{n+1} \cos (a)-i Q_{n-1} \sin (a) \\
& -i Q_{n+1} \sin (a)-i Q_{n+1} c^{2} \sin (a)+Q_{n-1} \cos (a)-i c^{2} Q_{n-1} \sin (a)+c^{2} Q_{n-1} \cos (a)=0 .
\end{aligned}
$$

For simplicity, let the wave number $a$ be $\pi / 2$. Representing each term $Q_{n}$ in the form $Q_{n}=Q 1_{n}+i Q 2_{n}$ allows us to separate the real and imaginary parts of Eq. (3.1), and write the system of real equations

$$
\begin{aligned}
& Q 1_{n, t}-2 c^{2} Q 2_{n}+c^{2} Q 2_{n-1}+Q 2_{n-1}+Q 2_{n+1}+c^{2} Q 2_{n+1}-2 Q 2_{n}=0, \\
& Q 2_{n, t}-c^{2} Q 1_{n-1}-c^{2} Q 1_{n+1}-2 c^{2} Q 1_{n}+2 Q 1_{n}-Q 1_{n-1}-Q 1_{n+1}=0 .
\end{aligned}
$$

The solutions of Eqs. (3.1) and (3.2) are sought in the form

$$
Q 1_{n}=F e^{g t+i k n}, \quad Q 2_{n}=G e^{g t+i k n},
$$

where $F, G$ are real constant amplitudes of the perturbation eigenmode, $g$ denotes the modulational instability gain, and $k$ the arbitrary real wave number of a small perturbation. Substituting Eq. (3.3) into Eq. (3.2) yields the dispersion relation for the perturbation viz. the condition for the existence of nontrivial solutions $F$ and $G$ :

or

$$
\left|\begin{array}{cc}
g & 2 \cos (k)+2 \cos (k) c^{2}-2 c^{2}-2 \\
-2 \cos (k) c^{2}-2 \cos (k)-2 c^{2}+2 & g
\end{array}\right|=0,
$$

$$
g^{2}=4\left(1+c^{2}\right)(1-\cos (k))\left(\cos (k) c^{2}+\cos (k)-1+c^{2}\right) .
$$

The modulational instability thus occurs if the right-hand side of (3.4) is positive - i.e. if $\cos (k)>\left(1-c^{2}\right)\left(1+c^{2}\right)$. Fig. 1 shows the gain $g(k)$ for $c=2,3$ and 5 at three power levels. Furthermore, at the point $\cos (k)=1 /\left(1+c^{2}\right)$ the function $g^{2}$ attains its maximum $g_{\max }^{2}=4 c^{4}$, so that the plane-wave states are subject to modulational instability and rogue waves can be solutions of Eq. (1.1). 


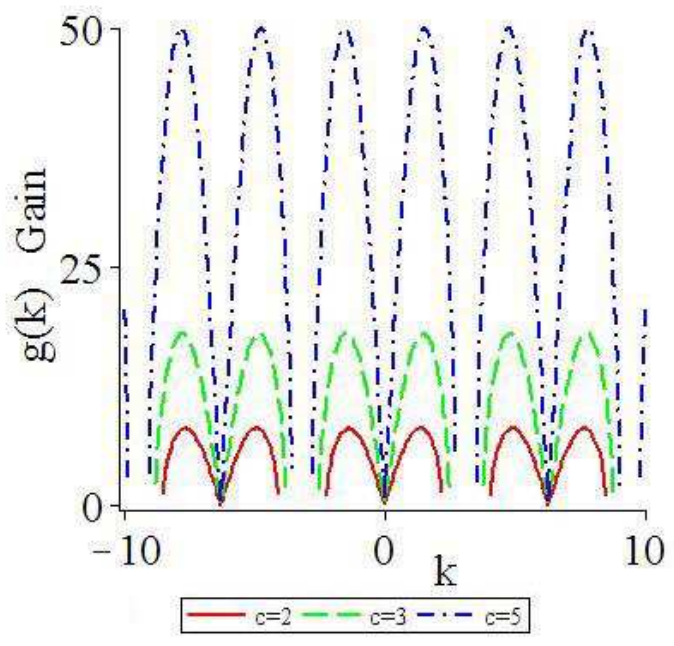

Figure 1: The gain spectra of modulation instability for various plane-wave amplitudes obtained from Eq. (2.9) and reflected in the positive growth rate $g(k)$. Different lines correspond to different plane-wave amplitudes: solid for $c=2$, dashed for $c=3$ and dot-dashed for $c=5$.

\section{Generalised Perturbation $(n, N-n)$-Fold Darboux Transformation}

Let us construct a generalised perturbation $(n, N-n)$-fold Darboux transformation for Eq. (1.1) based on Eqs. (1.2) and (1.3) via the gauge transformation

$$
\tilde{\varphi}_{n}=T_{n} \varphi_{n},
$$

with $\widetilde{\varphi}_{n}$ satisfying Eqs. (1.2)-(1.3) and $U_{n}$ and $V_{n}$ replaced by $\widetilde{U}_{n}$ and $\widetilde{V}_{n}$ such that

$$
\tilde{\varphi}_{n+1}=\widetilde{U}_{n} \widetilde{\varphi}_{n}, \quad \tilde{\varphi}_{n, t}=\widetilde{V}_{n} \widetilde{\varphi}_{n},
$$

where $\widetilde{U}_{n}=T_{n+1} U_{n} T_{n}{ }^{-1}$ and $\widetilde{V}_{n}=\left(T_{n, t}+T_{n} V_{n}\right) T_{n}{ }^{-1}$ are of the same form as $U_{n}$ and $V_{n}$ but the terms $u_{n}$ are everywhere replaced by $\widetilde{u}_{n}$. In order to guarantee the consistency of the Darboux transformation for Eq. (1.1), let us consider the transformation matrix

$$
T_{n}:=\left(\begin{array}{cc}
\lambda^{2 N}+\sum_{j=0}^{N-1} a_{n}^{(2 j)} \lambda^{2 j} & \sum_{j=0}^{N-1} b_{n}^{(2 j+1)} \lambda^{2 j+1} \\
-\sum_{j=0}^{N-1} b^{(2 N-2 j-1)^{*}} \lambda^{2 j+1} & 1+\sum_{j=0}^{N-1} a^{(2 N-2 j-2)^{*}} \lambda^{2 j+2}
\end{array}\right),
$$

where $N$ is a non-negative integer, and $a_{n}^{(2 j)}$ and $b_{n}^{(2 j+1)}(0 \leq j \leq N-1)$ are the solutions of the systems of linear algebraic equations $T\left(\lambda_{k}\right) \varphi_{k}\left(\lambda_{k}\right)=0(k=1,2, \cdots, N)$. The transformations (4.1)-(4.2) and $\widetilde{U}_{n}=T_{n+1} U_{n} T_{n}{ }^{-1}$ allow us to establish an important relation between the initial $u_{n}$ and new $\tilde{u}$ solutions and to show the form invariance of $\widetilde{U}_{n}, \widetilde{V}_{n}$ and $U_{n}, V_{n}$. This relation along with the procedure (4.1) is called the $N$-fold Darboux transformation of Eq. (1.1). The construction is similar to the corresponding constructions in 
Refs. $[12,37,38,40]$ but in order to make the paper self-contained, we note some results here.

Theorem 4.1. Let $\varphi_{i}\left(\lambda_{i}\right)=\left(\phi_{i}, \psi_{i}\right)^{T}(i=1,2, \cdots, N)$ be $N$ distinct column vector solutions of the corresponding spectral problem (1.2) and (1.3) for the spectral parameters $\lambda_{1}, \lambda_{2}, \cdots, \lambda_{N}$ and the initial solution $u_{n}$ of Eq. (1.1). Then the $N$-fold Darboux transformation of Eq. (1.1) is given by

$$
\widetilde{u}=b_{n+1}^{(1)}+u_{n} a_{n+1}^{(0)},
$$

with $a_{n}^{(0)}=\Delta a_{n}^{(0)} / \Delta_{N}, b_{n}^{(1)}=\Delta b_{n}^{(1)} / \Delta_{N}$,

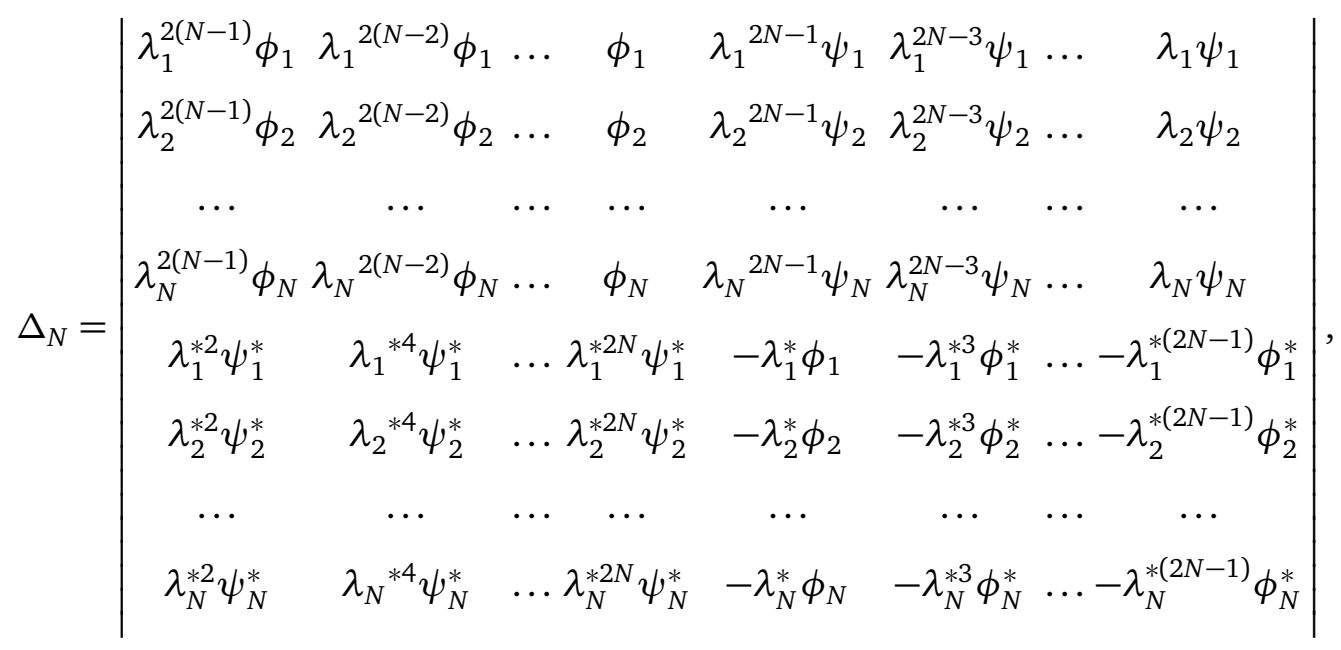

whereas $\Delta a_{n}^{(0)}$ and $\Delta b_{n}^{(1)}$ are obtained from the determinant $\Delta_{N}$ by, respectively, replacing the $N$-th and $2 N$-th columns with the column $\left(-\lambda_{1}^{2 N} \phi_{1},-\lambda_{2}^{2 N} \phi_{2}, \cdots,-\lambda_{N}^{2 N} \phi_{N},-\psi_{1}^{*},-\psi_{2}^{*}, \cdots\right.$, $\left.-\psi_{N}^{*}\right)^{\mathrm{T}}$.

Thus the $N$-fold Darboux transformation (4.3) maps the solution $u_{n}$ of Eq. (1.1) into a new solution $\widetilde{u}_{n}$. This transformation can be used to determine the multi-soliton solutions (or multi-breather solutions) of Eq. (1.1) when $u_{n}=0$ is the initial solution, although this is not the main goal here. Rather, let us modify the $N$-fold Darboux transformation in order to express the higher-order rogue wave and rational soliton solutions in terms of the determinants of Eq. (1.1). This can be done for $N$-fold Darboux transformations with $N$ distinct eigenvalues, but the number $n$ of the eigenvalues $\lambda$ must be changed. If $1 \leq n \leq N$, then the generalised perturbation $(n, N-n)$-fold Darboux transformation of Eq. (1.1) is described by the following theorem.

Theorem 4.2. Let $\varphi_{i}\left(\lambda_{i}\right)=\left(\phi_{i}\left(\lambda_{i}\right), \psi_{i}\left(\lambda_{i}\right)\right)^{\mathrm{T}}(i=1,2, \cdots, n)$ be column vector solutions of the Lax pairs (1.2) and (1.3) for the spectral parameters $\lambda_{i}(i=1,2, \cdots, n)$ and the same initial solution $u_{0}$ of Eq. (1.1). The generalised perturbation $(n, N-n)$-fold Darboux transformation of Eq. (1.1) given by

$$
\widetilde{u}=b_{n+1}^{(1)}+u_{0} a_{n+1}^{(0)},
$$


with $a_{n}^{(0)}=\Delta a_{n}^{(0)} / \Delta_{N}^{\epsilon(n)}, b_{n}^{(1)}=\Delta b_{n}^{(1)} / \Delta_{N}^{\epsilon(n)}, \Delta_{N}^{\epsilon(n)}=\operatorname{det}\left(\left[\Delta^{(1)} \cdots \Delta^{(n)}\right]^{\mathrm{T}}\right)$, $\Delta^{(i)}=\left(\Delta_{j, s}^{(i)}\right)_{2\left(m_{i}+1\right) \times 2 N}$ and $\Delta_{j, s}^{(i)}, 1 \leq j \leq 2\left(m_{i}+1\right), 1 \leq s \leq N, i=1,2, \cdots, n$ defined by $\Delta_{j, s}^{(i)}=$

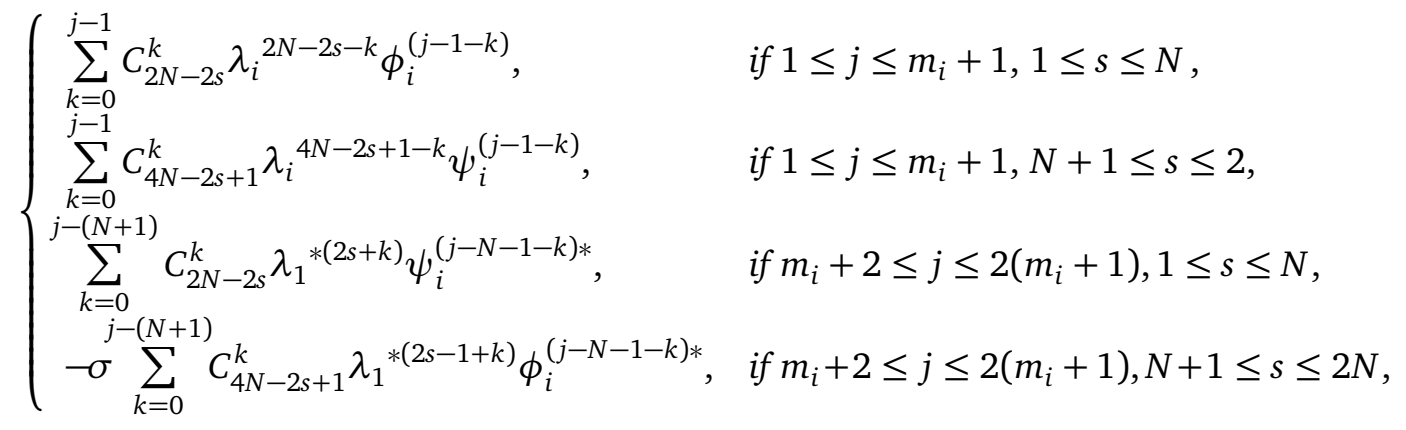

where $\Delta a_{n}^{(0)}$ and $\Delta b_{n}^{(1)}$ are obtained from the determinant $\Delta_{N}^{\epsilon(n)}$ by replacing the $N$-th and $(2 N)$-th column with the column vector $\left(b^{(1)} \cdots b^{(n)}\right)^{\mathrm{T}}, b^{(i)}=\left(b_{j}^{(i)}\right)_{2\left(m_{i}+1\right) \times 1}$ and

$$
b_{j}^{(i)}=\left\{\begin{array}{l}
-\sum_{k=0}^{j-1} C_{2 N}^{k} \lambda_{i}^{2 N-k} \phi_{i}^{(j-1-k)} \text { for } 1 \leq j \leq m_{i}+1, \\
-\psi_{i}^{(j-N-1) *} \text { for } m_{i}+2 \leq j \leq 2\left(m_{i}+1\right) .
\end{array}\right.
$$

Remark 4.1. Note that $n$ in the notation "(n,N-n)-fold" denotes the number of distinct eigenvalues or the order of the vector eigenfunction $\varphi_{n}$ and $N-n$ the sum of the orders of the highest derivative of the Darboux matrix $T$ in (4.2). If $n=N$ and $m_{i}=0,1 \leq i \leq N$, this transformation reduces to the $N$-fold Darboux transformation in Theorem 4.1. Indeed, the $N$-fold Darboux transformation is a special case of the generalised perturbation $(n, N-n)$ fold Darboux transformation. If $n=1$ and $m_{1}=N-1$, the corresponding transformation reduces to the generalised perturbation $(1, N-1)$-fold Darboux transformation, used in the next section to obtain higher-order rogue wave and rational soliton solutions of Eq. (1.1). If $n \neq 1$ or $n \neq N$, one can obtain other Darboux transformations, but they are not discussed here.

\section{Higher-Order Rogue Wave and Rational Soliton Solutions}

We employ the generalised perturbation $(1, N-1)$-fold Darboux transformation to study rogue wave and rational soliton solutions of Eq. (1.1) using the initial plane-wave solution $u_{0}=c e^{i\left(a n+\left(2+2 c^{2}\right) \sin (a) t\right)}$, where $c$ and $a$ are real-valued constants and $i=\sqrt{-1}$. If $a \neq 0$, the solutions of Eqs. (1.2) and (1.3) are very complicated, so let us consider two cases: - viz. a nonzero plane-wave background where $a=\pi / 2$ and a non-vanishing constant background where $a=0$ related to a discrete rogue wave and a discrete rational soliton, respectively. 


\subsection{Dynamical behaviour of higher-order rogue wave solutions for nonzero plane-wave background}

Let $a=\pi / 2$. On substituting the plane-wave solution $u_{0}=c e^{i\left((\pi / 2) n+\left(2+2 c^{2}\right) t\right)}$ into Eqs. (1.2)- (1.3) one obtains the solution

$$
\varphi(\lambda)=C_{1} \tau^{n} e^{\rho t+\Theta(\varepsilon)}\left(\begin{array}{c}
1 \\
\frac{i-\lambda^{2}+\sqrt{\lambda^{4}-2 i \lambda^{2}-4 i \lambda^{2} c^{2}-1}}{2 \lambda c} e^{-i\left((\pi / 2) n+\left(2+2 c^{2}\right) t\right)}
\end{array}\right),
$$

where

$$
\begin{aligned}
& \tau=\frac{i+\lambda^{2}+\sqrt{\lambda^{4}-2 i \lambda^{2}-4 i \lambda^{2} c^{2}-1}}{2} \\
& \Theta(\varepsilon)=\sqrt{\lambda^{4}-2 i \lambda^{2}-4 i \lambda^{2} c^{2}-1} \sum_{k=1}^{N}\left(b_{k}+d_{k} i\right) \varepsilon^{2 k} \\
& \rho=\frac{2 i \lambda^{2}+2 i \lambda^{2} c^{2}-\left(i-\lambda^{2}\right) \sqrt{\lambda^{4}-2 i \lambda^{2}-4 i \lambda^{2} c^{2}-1}}{2 \lambda^{2}},
\end{aligned}
$$

$C_{1}$ is an arbitrary constant, $\varepsilon$ is a small parameter and $b_{k}, d_{k}(k=1,2, \cdots, N)$ are free real parameters.

Let us represent $\lambda$ as $\lambda=\lambda_{1}+\varepsilon^{2}, \lambda_{1}=\sqrt{i\left(2 c^{2}+1+2 \sqrt{c^{2}\left(1+c^{2}\right)}\right)}$ and expand $\varphi$ in Taylor series around $\varepsilon=0$. To simplify the calculations, let us also set $c=3 / 4$ so that $\lambda_{1}=\sqrt{2}+i \sqrt{2}$, hence

$$
\varphi\left(\varepsilon^{2}\right)=\varphi^{(0)}+\varphi^{(1)} \varepsilon^{2}+\varphi^{(2)} \varepsilon^{4}+\varphi^{(3)} \varepsilon^{6}+\cdots
$$

where

$$
\varphi^{(0)}=\left(\begin{array}{c}
\phi^{(0)} \\
\psi^{(0)}
\end{array}\right)=\left(\begin{array}{c}
C_{1}\left(\frac{5 i}{2}\right)^{n} e^{\frac{25}{16} i t} \\
-C_{1} \frac{1+i}{2}\left(\frac{5 i}{2}\right)^{n} e^{-i\left(\frac{\pi}{2} n+\frac{25}{16} t\right)} \sqrt{2}
\end{array}\right)
$$

the components $\phi^{(i)}, \psi^{(i)}(i=1,2)$ of the coefficients $\varphi^{(1)}, \varphi^{(1)}$ of the series (5.1) are given in Appendix $\mathrm{A}$ and $C_{1}=1$.

Eqs. (4.1), (4.4) and (5.1) can now be used in order to derive new solutions of Eq. (1.1). For $N=1$ the seed solution $\widetilde{u}_{1}(n, t)=-c e^{i\left((\pi / 2) n+\left(2+2 c^{2}\right) t\right)}$ produces wave propagation defined by the nontrivial solutions (4.4) of Eq. (1.1) from different values of the parameter $N$ for the nonzero plane-wave background, with the corresponding wave structures for $N=2,3,4$ illustrated in Figs. 2-8.

Case I. If $N=2$, then according to Theorem 4.2 there is the first-order rogue wave solution of Eq. (1.1)

$$
\widetilde{u}_{2}(x, t)=b_{n+1}^{(1)}+u_{0} a_{n+1}^{(0)}
$$


with $b_{n}^{(1)}=\Delta b_{n}^{(1)} / \Delta_{2}^{\epsilon}, a_{2}^{(0)}=\Delta a_{n}^{(0)} / \Delta_{2}^{\epsilon}$ and

$$
\begin{array}{|cccc}
\Delta_{2}^{\epsilon}= & & & \\
\lambda^{2} \phi^{(0)} & \phi^{(0)} & \lambda^{3} \psi^{(0)} & \lambda \psi^{(0)} \\
\lambda^{2} \phi^{(1)}+2 \lambda \phi^{(0)} & \phi^{(1)} & \lambda^{3} \psi^{(1)}+3 \lambda^{2} \psi^{(0)} & \lambda \psi^{(1)}+\psi^{(0)} \\
\lambda^{* 2} \psi^{(0)^{*}} & \lambda^{* 4} \psi^{(0)^{*}} & -\lambda^{*} \phi^{(0)^{*}} & -\lambda^{* 3} \phi^{(0)^{*}} \\
\lambda^{* 2} \psi^{(1)^{*}}+2 \lambda^{*} \psi^{(0)^{*}} & \lambda^{* 4} \psi^{(1)^{*}}+4 \lambda^{* 3} \psi^{(0)^{*}} & -\lambda^{*} \phi^{(1)^{*}}-\phi^{(0)^{*}} & -\lambda^{* 3} \phi^{(1)^{*}}-3 \lambda^{* 2} \phi^{(0)^{*}}
\end{array} \mid,
$$

$\Delta a_{n}^{(1)}=$

$$
\left|\begin{array}{cccc}
\lambda^{2} \phi^{(0)} & -\lambda^{4} \phi^{(0)} & \lambda^{3} \psi^{(0)} & \lambda \psi^{(0)} \\
\lambda^{2} \phi^{(1)}+2 \lambda \phi^{(0)} & -\lambda^{4} \phi^{(1)}-4 \lambda^{3} \phi^{(0)} & \lambda^{3} \psi^{(1)}+3 \lambda^{2} \psi^{(0)} & \lambda \psi^{(1)}+\psi^{(0)} \\
\lambda^{* 2} \psi^{(0)^{*}} & -\psi^{(0)^{*}} & -\lambda^{*} \phi^{(0)^{*}} & -\lambda^{* 3} \phi^{(0)^{*}} \\
\lambda^{* 2} \psi^{(1)^{*}}+2 \lambda^{*} \psi^{(0)^{*}} & -\psi^{(1)^{*}} & -\lambda^{*} \phi^{(1)^{*}}-\phi^{(0)^{*}} & -\lambda^{* 3} \phi^{(1)^{*}}-3 \lambda^{* 2} \phi^{(0)^{*}}
\end{array}\right|,
$$

$\Delta b_{n}^{(1)}=$

$$
\left|\begin{array}{cccc}
\lambda^{2} \phi^{(0)} & \phi^{(0)} & \lambda^{3} \psi^{(0)} & -\lambda^{4} \phi^{(0)} \\
\lambda^{2} \phi^{(1)}+2 \lambda \phi^{(0)} & \phi^{(1)} & \lambda^{3} \psi^{(1)}+3 \lambda^{2} \psi^{(0)} & -\lambda^{4} \phi^{(1)}-4 \lambda^{3} \phi^{(0)} \\
\lambda^{* 2} \psi^{(0)^{*}} & \lambda^{* 4} \psi^{(0)^{*}} & -\lambda^{*} \phi^{(0)^{*}} & -\psi^{(0)^{*}} \\
\lambda^{* 2} \psi^{(1)^{*}}+2 \lambda^{*} \psi^{(0)^{*}} & \lambda^{* 4} \psi^{(1)^{*}}+4 \lambda^{* 3} \psi^{(0)^{*}} & -\lambda^{*} \phi^{(1)^{*}}-\phi^{(0)^{*}} & -\psi^{(1)^{*}}
\end{array}\right|
$$

Using symbolic computation, the expression for (5.2) can be simplified to provide the solution

$$
\widetilde{u}_{2}(n, t)=\frac{3}{4}\left(-1+\frac{1600+3600 i t}{576(n-1 / 3)^{2}+2025 t^{2}+256}\right) e^{i((\pi / 2) n+(25 / 8) t)}
$$

which has no singular points. To investigate the behaviour of this rogue wave solution, let us assume that $n \in(-\infty,+\infty)$ is a continuous variable. Fig. 2 displays the profile of rogue wave solution (5.3) around the point $(n, t)=(-4 / 3,0)$, with $\left|\widetilde{u}_{2}(n, t)\right| \longrightarrow$ $\left|\widetilde{u}_{2, \infty}(n, t)\right|=3 / 4$ (background) as $n, t \longrightarrow \infty$. The function $\left|\widetilde{u}_{2}(n, t)\right|$ has maximum value $\left|\tilde{u}_{2, \max }(n, t)\right|=63 / 16$ at the point $(n, t)=(-4 / 3,0)$ so $\left|\tilde{u}_{2, \max }(n, t)\right|=5.25\left|\tilde{u}_{2, \infty}(n, t)\right|$. Moreover, there is the conservation law

$$
\int_{-\infty}^{+\infty}\left(\left|\widetilde{u}_{2}(n, t)\right|^{2}-\frac{9}{16}\right) d n=0 .
$$

To study the dynamical behaviour of the first rogue wave solution (5.3), let us consider the intensity of $\widetilde{u}_{2}(n, t)$ - i.e.

$$
\left|\widetilde{u}_{2}(n, t)\right|^{2}=\frac{9}{16} \frac{\left(384 n+1280-576 n^{2}-2025 t^{2}\right)^{2}+3600^{2} t^{2}}{\left[576(n-1 / 3)^{2}+2025 t^{2}+256\right]^{2}},
$$


(a1)

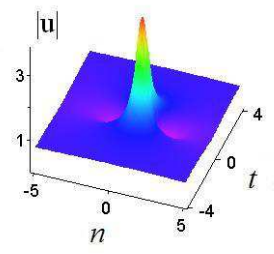

(b1)

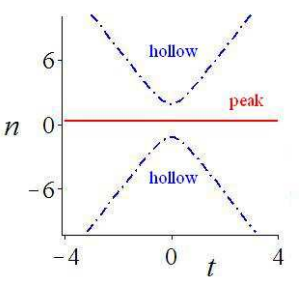

(c1)

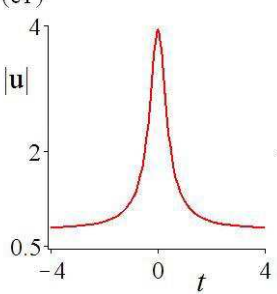

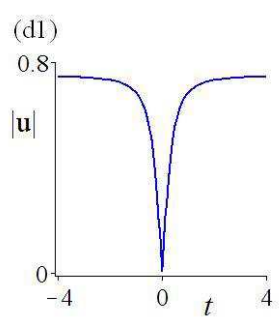

Figure 2: (a1) The first-order rogue wave solution (5.3), which has a peak and two depression points. (b1) The motion of the peak and hollow centres described by Eqs. (5.5)-(5.6). (c1) The evolution of the absolute value of the field at the peak point $(n=1 / 3)$. (d1) The evolution of the hollow points $\left(n=1 / 3 \pm(1 / 24) \sqrt{1344+6075 t^{2}}\right)$.

which has three critical points $\left(n_{1}, t_{1}\right)=(1 / 3,0),\left(n_{2,3}, t_{2,3}\right)=((1 \pm \sqrt{21}) / 3,0)$. The critical point $\left(n_{1}, t_{1}\right)$ is the maximum point where the maximum amplitude is $63 / 16$. The other critical points $\left(n_{2,3}, t_{2,3}\right)$ are minimum (depression) points, such that the minimum amplitude is zero.

For the intensity (5.4), the trajectory of the peak centres depends on the behaviour of its spatial coordinate $T_{h}=T_{h}(t)$. It follows from Eq. (5.4) that

$$
T_{h}=\frac{1}{3}
$$

and for the centres of the depression points the spatial coordinates $T_{c \pm}$ are

$$
T_{c \pm}=\frac{1}{3} \pm \frac{1}{24} \sqrt{1344+6075 t^{2}} .
$$

This allows us to determine the rogue wave width $T_{d}$ defined as the spatial distance between the two depression points,

$$
T_{d}=\frac{1}{12} \sqrt{1344+6075 t^{2}} .
$$

Let $\alpha, \beta$ be real numbers. If the transformations $n=n+\alpha+1 / 3, t=t+\beta$ are applied to the rogue wave solution (5.3), then one obtains the rogue wave solution

$$
\widetilde{u}_{2}=\frac{3}{4}\left[\frac{1600+3600 i(t+\beta)}{576(n+\alpha)^{2}+2025(t+\beta)^{2}+256}-1\right] e^{i\left(\frac{\pi}{2}\left(n+\alpha-\frac{1}{3}\right)+\frac{25}{8}(t+\beta)\right)} .
$$

The rogue wave $\widetilde{u}_{2}=\widetilde{u}_{2}(n, t)$ depends on $\alpha$ and $\beta$, hence the position of the corresponding peak can be controlled by changing these parameters. Fig. 3 shows the rogue waves with different parameters $\alpha, \beta$. The amplitude $c$ of the plane-wave background can be used to control the first-order rogue wave solution (5.2), and here for example the parameter $c$ modulates its shape, centre, and amplitude.

- If $c=5 / 12$, i.e. if $\lambda=(3 \sqrt{2}+3 \sqrt{2} i) / 4$, the solution $u_{2}(n, t)$ peaks around the point $(4 / 5,0)$, and the background value $c=5 / 12$ is the limit of $\left|u_{2}(n, t)\right|$ as $n, t$ tend to $\infty$. The maximum value of $\left|u_{2}(n, t)\right|$ is $665 / 432$ attained at the point $(4 / 5,0)$, and the minimum is zero achieved at $((4 \pm \sqrt{133}) / 5,0)$. 

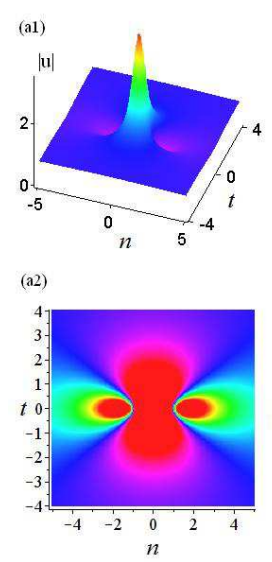

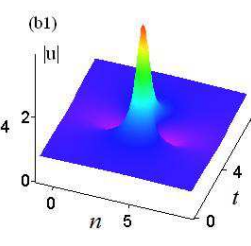

(b2)

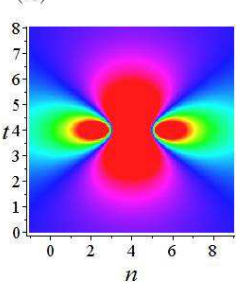

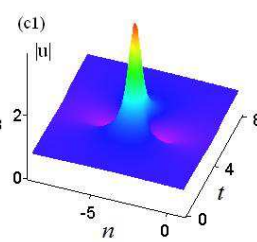

(c2)

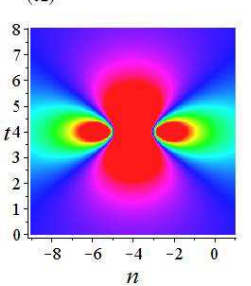

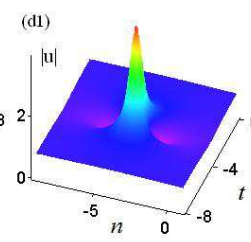
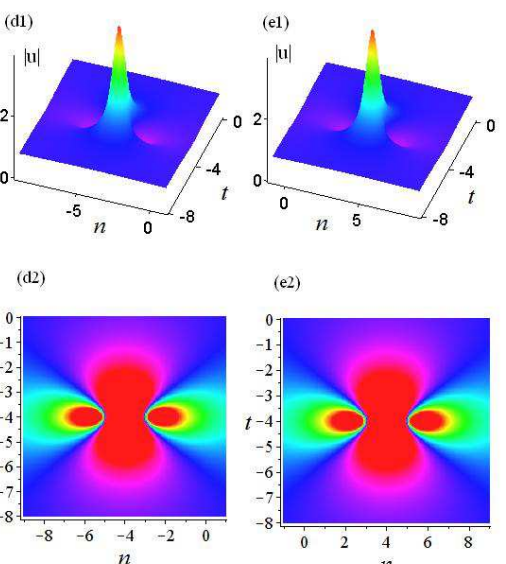

(e2)

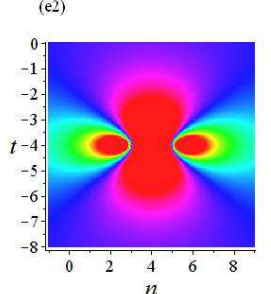

Figure 3: The first-order rogue wave solution $\tilde{u}_{2}(n, t)$ of Eq. (5.7) for various parameters $\alpha$ and $\beta$. (a) $\alpha=0, \beta=0$ : the peak centre is located at $(n, t)=(0,0)$. (b) $\alpha=-4, \beta=-4$ : the peak centre is located at $(n, t)=(4,4)$. (c) $\alpha=4, \beta=-4$ : the peak centre is located at $(n, t)=(-4,4)$. (d) $\alpha=4$, $\beta=4$ : the peak centre is located at $(n, t)=(-4,-4)$. (e) $\alpha=-4, \beta=4$ : the peak centre is located at $(n, t)=(4,-4)$.

- If $c=4 / 3$, i.e. if $\lambda=(3 \sqrt{2}+3 \sqrt{2} i) / 2$, the solution $u_{2}(n, t)$ peaks around the point $(1 / 8,0)$, and the background value $c=4 / 3$ is the limit of $\left|u_{2}(n, t)\right|$ as $n, t$ tend to $\infty$. The maximum value of $\left|u_{2}(n, t)\right|$ is $364 / 27$ attained at the point $(1 / 8,0)$, and the minimum is zero achieved at $((1 \pm \sqrt{91}) / 8,0)$.

- If $c=12 / 5$, i.e. if $\lambda=(5 \sqrt{2}+5 \sqrt{2} i) / 2$, the solution $u_{2}(n, t)$ peaks around the point $(1 / 24,0)$, and the background value $c=12 / 5$ is the limit of $\left|u_{2}(n, t)\right|$ as $n, t$ tend to $\infty$. The maximum value of $\left|u_{2}(n, t)\right|$ is $7812 / 125$ attained at the point $(1 / 24,0)$, and the minimum is zero achieved at $((1+\sqrt{651}) / 24,0)$.

Thus the increase of the spectral parameter $|\lambda|$ is related to the increase of $c$, and the amplitude of the first-order rogue wave also grows. The centre and the peak of the firstorder rogue wave move to the right along the line $t=0$.

The results of the numerical simulations for the propagation of the discrete first-order rogue wave (5.3) are presented in Fig. 4, showing both the exact solution and that perturbed by a $2 \%$ noise. During the time $t \in(-2,2)$, the time evolution profile of the rogue wave (5.3) with no noise is almost the same as of the exact rogue wave. However, the noise added to the initial solution leads to a weak oscillation at a later time.

Case II. According to Theorem 4.2, for $N=3$ the second-order rogue wave solution is

$$
\widetilde{u}_{3}(n, t)=b_{n+1}^{(1)}+u_{0} a_{n+1}^{(0)} .
$$

The analytic expression of this third-order solution is very cumbersome, and so not given it here. However, the second-order rogue wave solution profile is displayed in Fig. 5. The parameters $b_{1}$ and $d_{1}$ in (5.8) can be used to split the second-order rogue wave into three first-order rogue waves, with centre points forming a triangle as shown in Fig. 5, graphs 
(a)

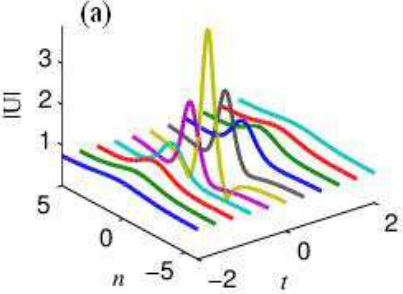

(b)

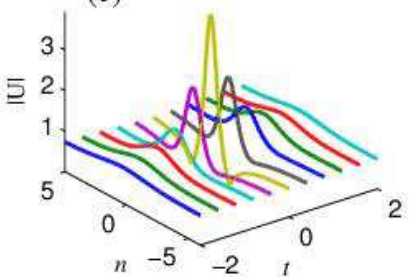

(c)

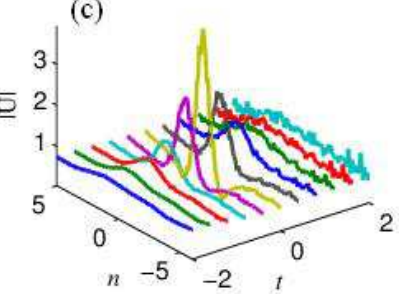

Figure 4: The first-order rogue wave solution (5.3). (a) Exact solution. (b) Time evolution of the exact solution (5.3). (c) Time evolution of the exact solution (5.3) perturbed by a $2 \%$ noise.

(a1)

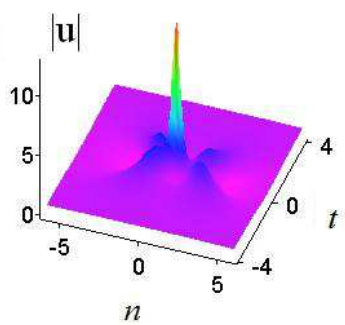

(a2)

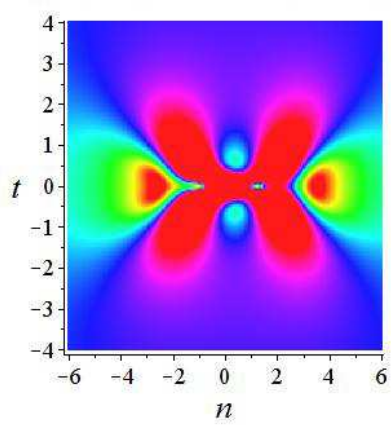

(b1)

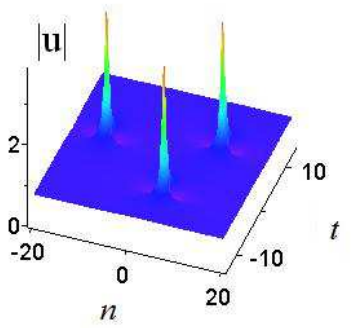

(b2)

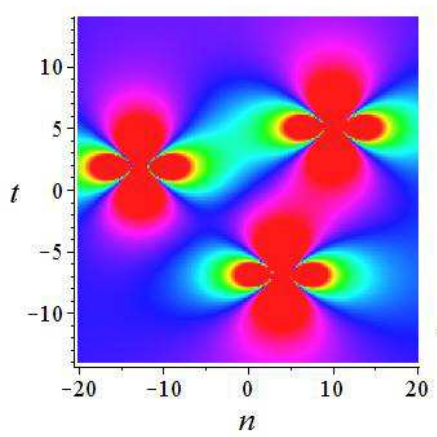

(c1)

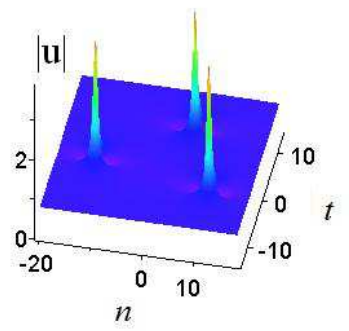

(c2)

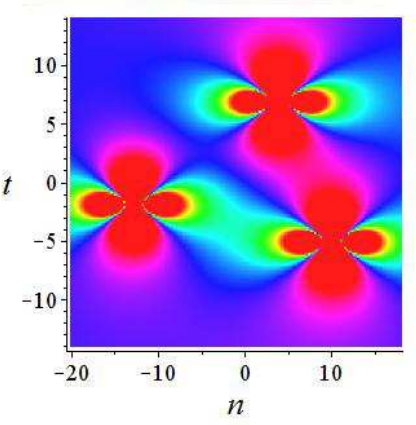

Figure 5: The second-order rogue wave solution Eq. (5.8). (a1)-(a2): Strong interaction with higher amplitude $b_{1}=d_{1}=0$; (b1)-(b2): Weak interaction with lower amplitude $b_{1}=100, d_{1}=0$. (c1)-(c2): Weak interaction with lower amplitude $b_{1}=0, d_{1}=100$.

(b1)-(b2) and (c1)-(c2). It is notable that the sides of this triangle grow with increasing $\left|b_{1}\right|$ and $\left|d_{1}\right|$. Moreover, the parameter $d_{1}$ can also control the rotation of the rogue wave profile. Similar to the first-order rogue wave, the transformations $n=n+\alpha, t=t+\beta$ applied to Eq. (5.8) provide a controllable second-order rogue wave solution.

Numerical simulations again allow us to study the behaviour of the second-order rogue wave solution (5.8). Let us consider the strong and weak interaction cases presented in Fig. 5 graphs (a) and (b), respectively. For the strong interaction, the profile of the secondorder rogue wave solution (5.8) with no noise remains almost the same as the exact solution 

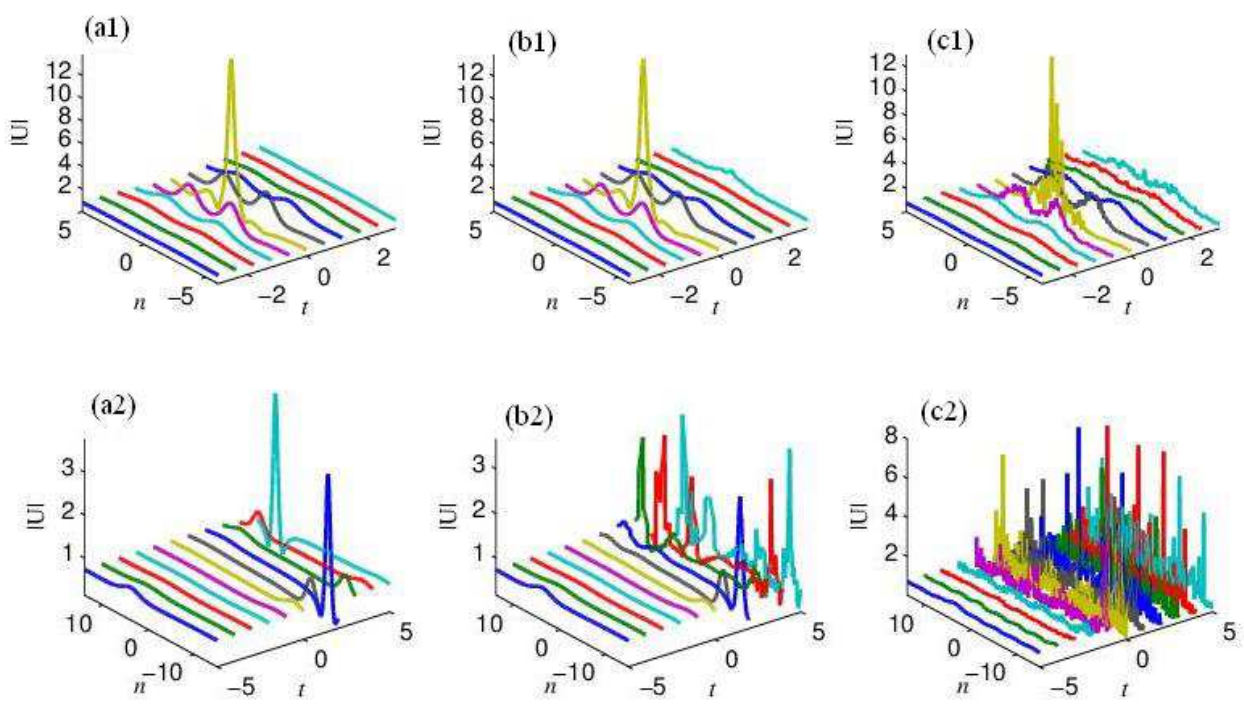

Figure 6: The second-order rogue wave solution (5.8). Exact solutions with (a1) $b_{1,2}=d_{1,2}=0$ and (a2) $b_{1}=100, d_{1}=0$, (b1)-(b2) Time evolution using exact solution (5.8) as initial conditions, and (c1)-(c2) time evolution using exact solution (5.8) perturbed by a $2 \%$ noise as initial conditions.

(5.8) during the time period $(-2,2)$. However, even a small noise causes oscillations at a later time - cf. Fig. 6 (c1). It can be seen that strong interaction exhibits a better propagation stability. For the weak interaction case, the rogue wave solution (5.8) with $b_{1}=100$, $d_{1}=0$, the results are different. The second-order rogue wave solution (5.8) with no noise produces strong oscillations after $t=0-\mathrm{cf}$. Fig. 6 (b2). In the weak interaction case, when a small (2\%) noise is added the wave propagation has strong oscillations after $t=-1-$ cf. Fig. 6 (c2). Thus Fig. 6 (c1) and (c2) show that small noise has a weak (strong) effect in the strong (weak) interaction case. This can be due to energy focused close to the origin of the $(n, t)$-space in the strong interaction case, whereas in the weak interaction case it is spread around three points - cf. Fig. 6, graphs (a1) and (a2).

Case III. According to Theorem 4.2, if $N=4$ there is the third-order rogue wave solution

$$
\widetilde{u}_{4}(n, t)=b_{n+1}^{(1)}+u_{0} a_{n+1}^{(0)} \text {. }
$$

The analytical expression of the third-order solution (5.9) with parameters $C_{1}, b_{1}, b_{2}, d_{1}$ and $d_{2}$ is also cumbersome and not presented here. However, applying the transformation $n=n+\alpha, t=t+\beta$ to (5.9), one can again obtain a controllable third-order rogue wave solution with notable properties as follows.

- For $b_{1}=100, b_{2}=d_{1,2}=0$, the weak interaction of the third-order rogue wave splits in six first-order rogue waves, creating a triangular structure.

- For $b_{2}=10^{3}, b_{1}=d_{1,2}=0$, the weak interaction of the third-order rogue wave also splits into six first-order rogue waves, creating a pentagonal structure with a first-order rogue wave located almost in the centre of the pentagon. 
(a1)

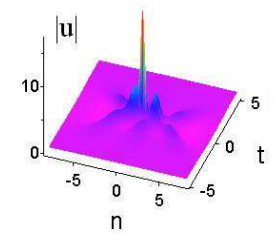

(a2)

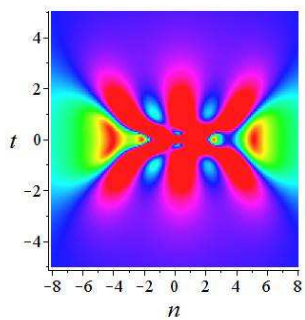

(b1)

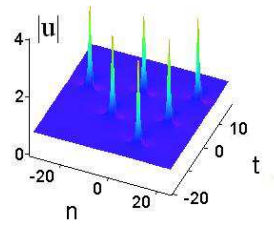

(b2)

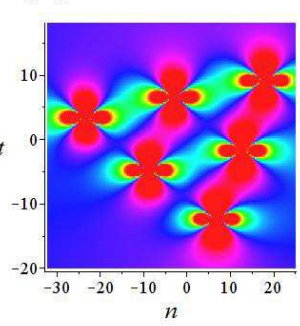

(c1)

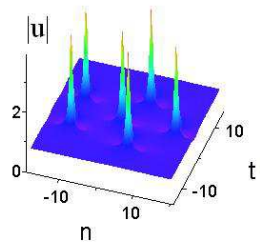

(c2)

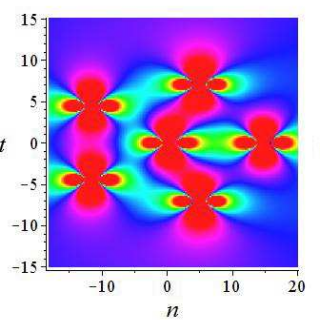

(d1)

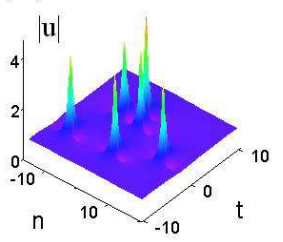

(d2)

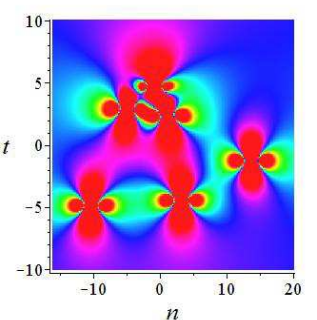

Figure 7: The third-order rogue wave solution Eq. (5.9). (a1)-(a2): Strong interaction $b_{1}=d_{1}=b_{2}=$ $d_{2}=0$. (b1)-(b2): Weak interaction $b_{1}=100, d_{1}=b_{2}=d_{2}=0$. (c1)-(c2): Weak interaction $b_{2}=1000$, $b_{1}=d_{1}=d_{2}=0$. (d1)-(d2): Weak interaction $b_{1}=-14, b_{2}=230, d_{1}=d_{2}=0$.

- If only two of the parameters $b_{1}, b_{2}, d_{1}, d_{2}$ are zero, then the third-order rogue wave solution (5.9) loses its symmetric structure.

Numerical simulations again illustrate the dynamical behaviour of the third-order rogue wave solution (5.9) in a strong and two weak interaction cases. For a short time it is notable that the wave propagation of the third-order rogue wave solution (5.9) with no noise is almost identical to the corresponding exact third-order rogue wave solution in each case. However, for the weak interaction the third-order rogue wave (5.9) with no noise is subject to strong oscillations after $t=5-$ cf. Fig. 8 (b3). If a small noise is added to the initial solutions, then for the strong interaction the wave propagation of the third-order rogue wave solution (5.9) is subject to weak oscillations - cf. Fig. 8 (c1), whereas wave propagations with weak interaction is subject to strong oscillations - cf. Fig. 8 (c2) and (c3).

\subsection{Dynamical behaviour of higher-order rational soliton solutions on non-vanishing constant background}

A constant solution $u_{0}=c$ of Eq. (1.1) on a non-vanishing constant background is now discussed. Recalling that such a solution appears when $a=0$, one may substitute $u_{0}=c$ into Eqs. (1.2) and (1.3) to obtain

$$
\varphi(\lambda)=C_{1} \tau^{n} e^{\rho t+\Theta(\varepsilon)}\left[\begin{array}{c}
1 \\
\frac{1-\lambda^{2}+\sqrt{1-2 \lambda^{2}+\lambda^{4}-4 \lambda^{2} c^{2}}}{2 \lambda c}
\end{array}\right]
$$



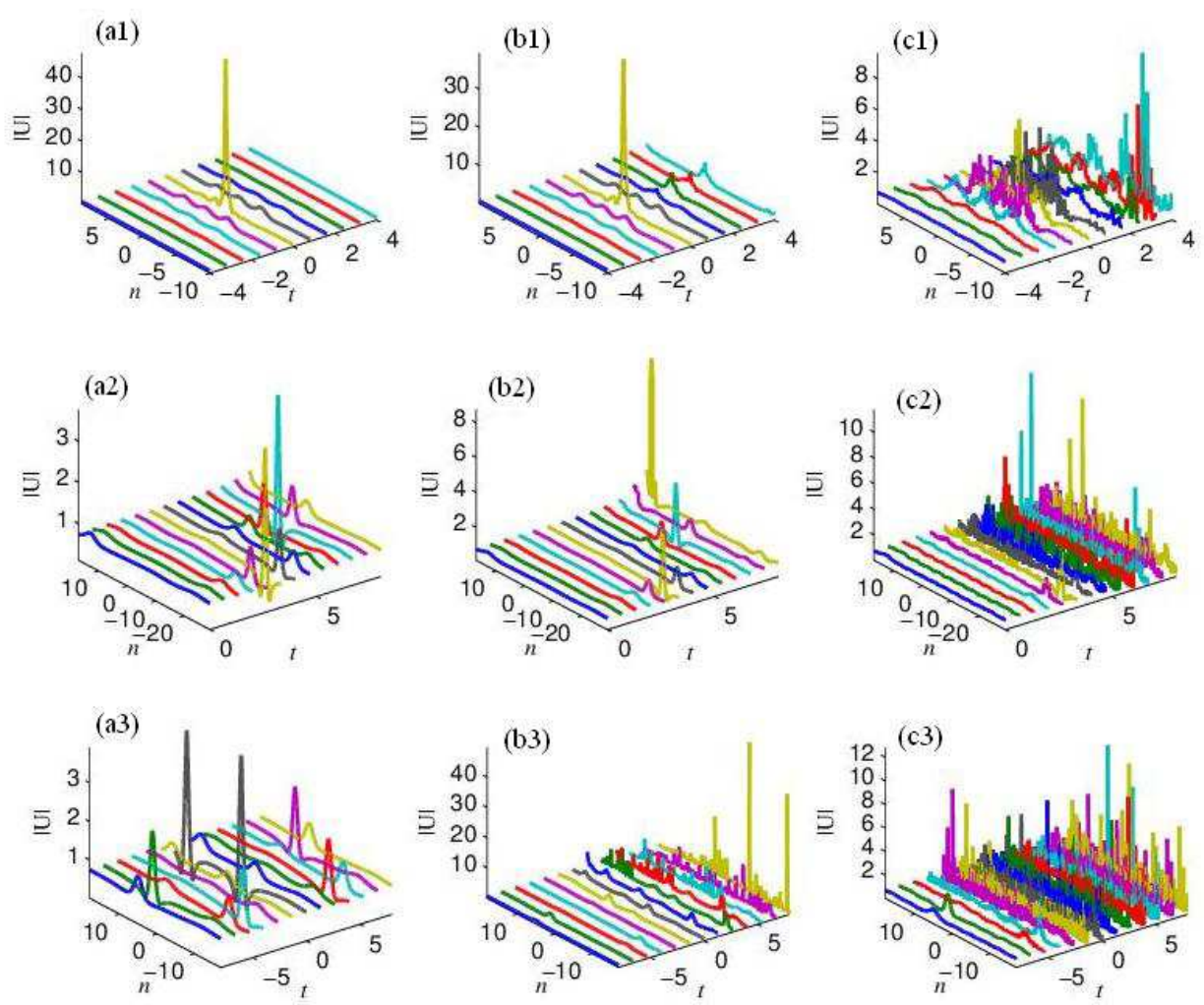

Figure 8: The third-order rogue wave solution (5.9). Left: Exact solution. Middle: Time evolution without noise. Right: Time evolution with a $2 \%$ noise. (a1)-(c1): $b_{1}=d_{1}=b_{2}=d_{2}=0 ;$ (a2)-(c2): $b_{1}=100, d_{1}=b_{2}=d_{2}=0$; (a3)-(c3): $b_{2}=1000, b_{1}=d_{1}=d_{2}=0$.

where

$$
\begin{aligned}
& \tau=\frac{1+\lambda^{2}+\sqrt{1-2 \lambda^{2}+\lambda^{4}-4 \lambda^{2} c^{2}}}{2}, \\
& \rho=\frac{2 \lambda^{2} c^{2}+\left(1+\lambda^{2}\right) \sqrt{1-2 \lambda^{2}+\lambda^{4}-4 \lambda^{2} c^{2}}}{2 \lambda^{2}}, \\
& \Theta(\varepsilon)=\sqrt{\lambda^{4}-2 i \lambda^{2}-4 i \lambda^{2} c^{2}-1} \sum_{k=1}^{N}\left(b_{k}+d_{k} i\right) \varepsilon^{2 k},
\end{aligned}
$$

with $C_{1}$ an arbitrary constant, $b_{k}, d_{k}(k=1,2, \cdots, N)$ real free parameters, and $\varepsilon$ a small parameter. Let us represent the spectral parameter in the form $\lambda=\lambda_{1}+\varepsilon^{2}$ where $\lambda_{1}=$ $-c+\sqrt{c^{2}+1}$, and expand the vector function $\varphi$ into two Taylor series around $\varepsilon=0$. To simplify the computation, let us set $c=3 / 4$ so $\lambda_{1}=1 / 2$ such that

$$
\varphi\left(\varepsilon^{2}\right)=\varphi^{(0)}+\varphi^{(1)} \varepsilon^{2}+\varphi^{(2)} \varepsilon^{2}+\varphi^{(3)} \varepsilon^{2}+\cdots,
$$


(a)

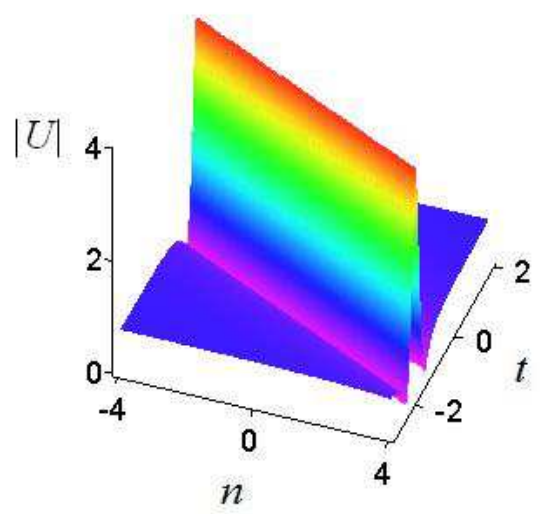

(b)

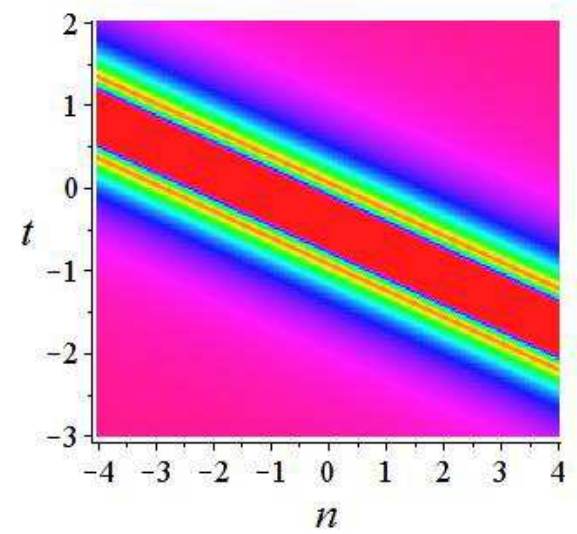

Figure 9: The first-order rational soliton solution $\tilde{u}_{2}(n, t)$ given by Eq. $(5.13)$.

where

$$
\varphi^{(0)}=\left(\begin{array}{l}
\phi^{(0)} \\
\psi^{(0)}
\end{array}\right)=\left(\begin{array}{l}
C_{1}\left(\frac{5}{8}\right)^{n} e^{\frac{9}{16} t} \\
C_{1}\left(\frac{5}{8}\right)^{n} e^{\frac{9}{16} t}
\end{array}\right),
$$

and $C_{1}=1$ in the resulting computations.

New solutions of Eq. (1.1) can now be found from the Eqs. (4.1), (4.4), (5.10) and (5.11). Figs. 9-14 show the propagation of the nontrivial solution (4.4) of Eq. (1.1) for $N=2,3,4$.

Case I. According to Theorem 4.2, for $N=2$ the first-order rational soliton solution is

$$
\widetilde{u}_{2}(x, t)=b_{n+1}^{(1)}+u_{0} a_{n+1}^{(0)},
$$

with $b_{n}^{(1)}=\Delta b_{n}^{(1)} / \Delta_{2}^{\epsilon}, a_{2}^{(0)}=\Delta a_{n}^{(0)} / \Delta_{2}^{\epsilon}$, and the expressions $\Delta_{2}^{\epsilon}, \Delta a_{n}^{(0)}, \Delta b_{n}^{(1)}$ are defined in the description of (5.2). Symbolic computations may be used to rewrite Eq. (5.12) as

$$
\widetilde{u}_{2}(n, t)=\frac{3}{4}\left[1-\frac{1600}{(24 n+75 t+32)^{2}+256}\right],
$$

which does not have any singular points.

Let us again assume that $n \in(-\infty, \infty)$ is a continuous variable. Fig. 9 shows the rational soliton solution (5.13). The peak of this soliton is located along the line $24 n+$ $75 t+32=0$, and its numerically obtained maximal amplitude is 3.9375 . The parameter $c$ can control the first-order rational soliton solution (5.12), and some solution properties related to $c$ are as follows.

- If $|c|$ increases, then the spectral parameter $|\lambda|$ increases and the amplitude of the first-order rational soliton grows. 
(a)

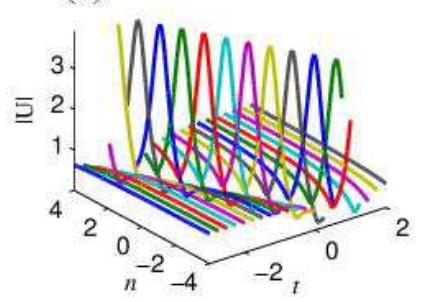

(b)

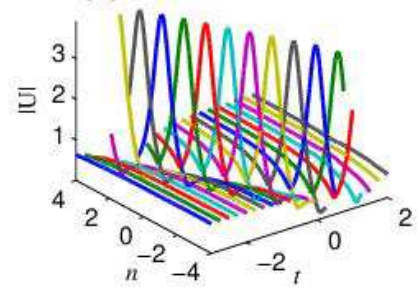

(c)

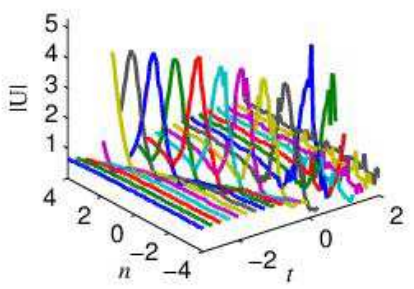

Figure 10: The first-order rational soliton solution (5.13). (a) Exact solution. (b) Evolution of the solution (5.13). (c) Evolution of the solution (5.13) perturbed by a $2 \%$ noise.

(al)

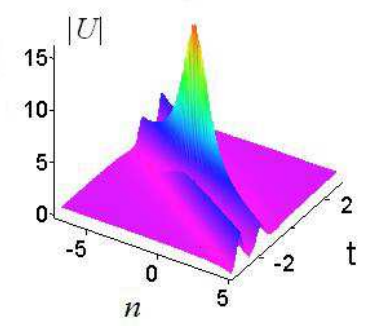

(a2)

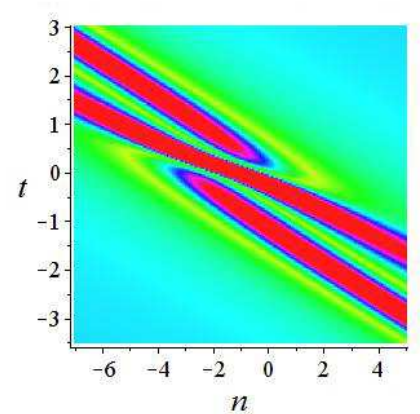

(b1)

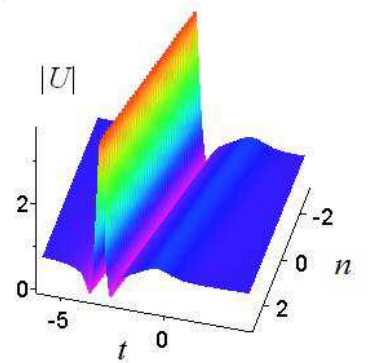

(b2)

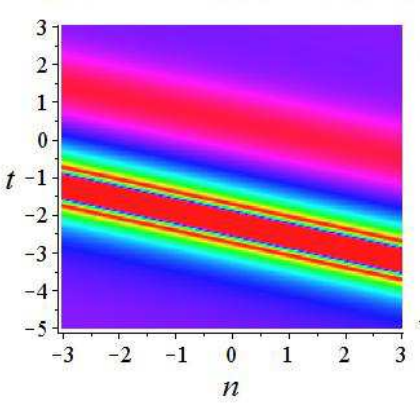

(c1)

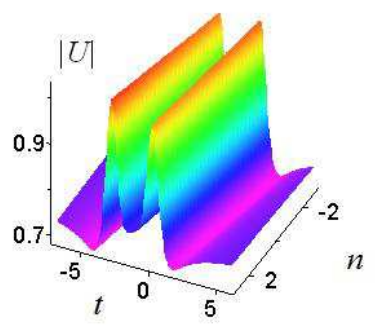

(c2)

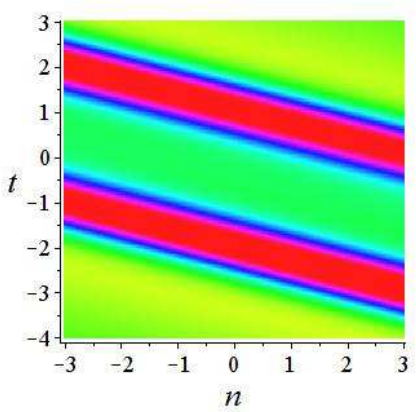

Figure 11: The second-order rational soliton solution Eq. (5.14). (a1)-(a2): Strong interaction with higher amplitude, $b_{1}=d_{1}=0$. (b1)-(b2): Weak interaction with lower amplitude, $b_{1}=100, d_{1}=0$. (c1)-(c2): Weak interaction with lower amplitude, $b_{1}=0, d_{1}=100$.

- If $|c| \rightarrow 0$ or $|c| \rightarrow \infty$ the first-order rational soliton transforms into a time or space soliton, respectively.

One may again use numerical simulations to explore dynamical behaviour of discrete firstorder rational soliton solutions. Fig. 10 shows the exact first-order rational soliton solution (5.13) of Eq. (1.1) and its evolution under various conditions. Thus in the absence of noise the rational soliton is not changing much during the time $t \in(-3,2)$, but if a small noise is added the wave has weak oscillations at the time $t=2$.

Case II. According to Theorem 4.2, for $N=3$ there is the second-order rational soliton 
(a1)

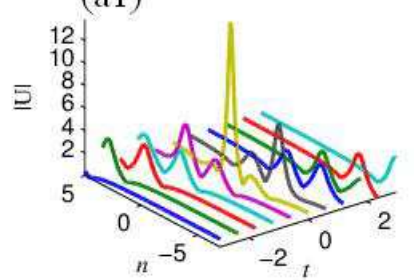

(a2)

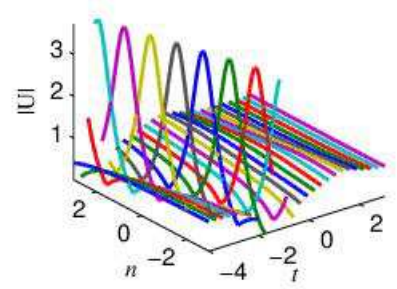

(a3)

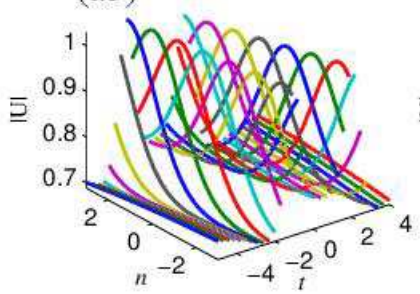

(b1)

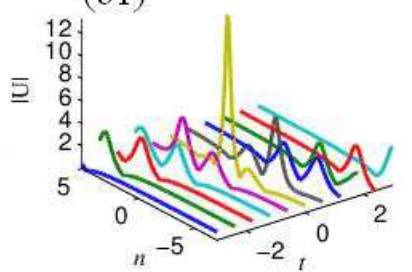

(b2)

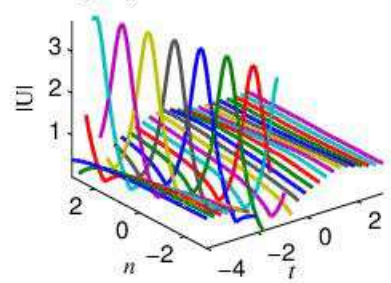

(b3)

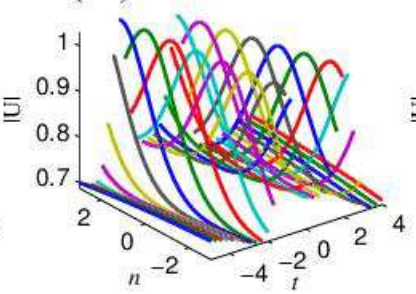

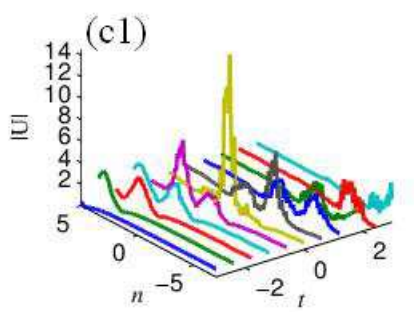

(c2)

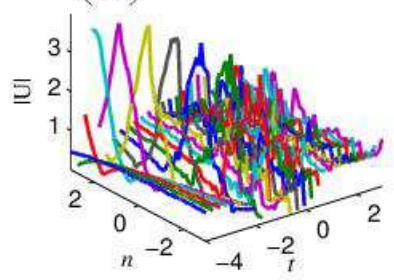

(c3)

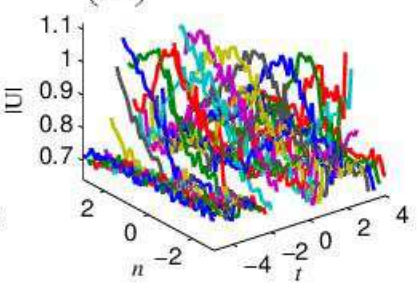

Figure 12: The second-order rational soliton solution (5.14). Exact solution: (a1) For $b_{1,2}=d_{1,2}=0$. (a2) For $b_{1}=100, d_{1}=0$. (a3) For $b_{1}=0, d_{1}=100$. (b1)-(b2): Evolution of exact solution. (c1)-(c2): Evolution of exact solution perturbed by a $2 \%$ noise.

solution

$$
\widetilde{u}_{3}(n, t)=b_{n+1}^{(1)}+u_{0} a_{n+1}^{(0)} .
$$

Although the analytic expression of this solution is not given here, its profile is displayed in Fig. 11. Similar to previous discussion, the parameters $b_{1}$ and $d_{1}$ can be used to represent the second-order rational soliton as three first-order rational solitons - cf. Fig. 11 (b1),(b2) and (c1), (c2). In particular, these parameters influence the shape and profile of the rational soliton.

Let us now consider the behaviour of the second-order rational soliton solution $\widetilde{u}_{3}(n, t)$ for strong and weak interactions. Similar to the second-order rogue wave, the strong interaction leads to a weak oscillation and better stability of the soliton solution, while the weak interaction produces a strong oscillation and instability - cf. Fig. 12.

Case III. According to Theorem 4.2, for $N=4$ there is the third-order rational soliton solution

$$
\widetilde{u}_{4}(n, t)=b_{n+1}^{(1)}+u_{0} a_{n+1}^{(0)} .
$$

We again do not give the analytic expression of this solution, but find that the choice of parameters $C_{1}, b_{1}, b_{2}, d_{1}, d_{2}$ influence the structure of the third-order rational soliton 
(a1)

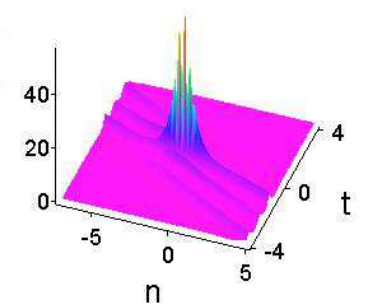

(a2)

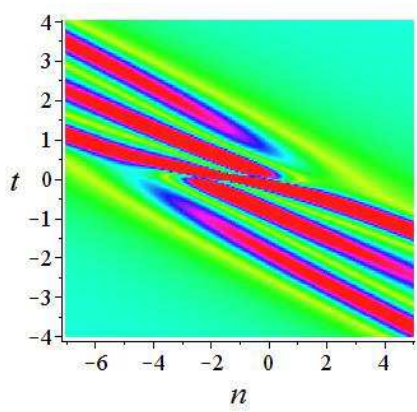

(b1)

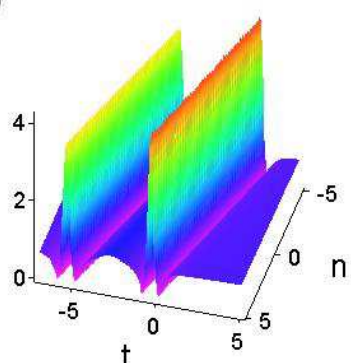

(b2)

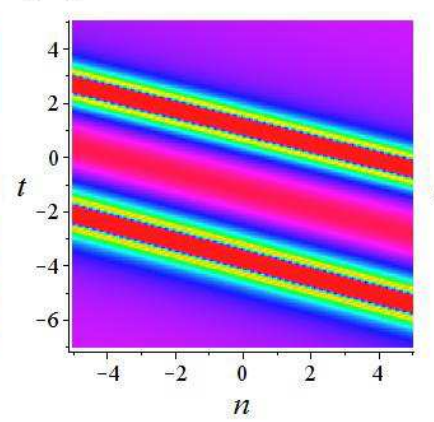

(c1)

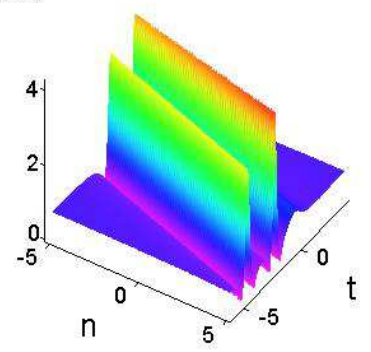

(c2)

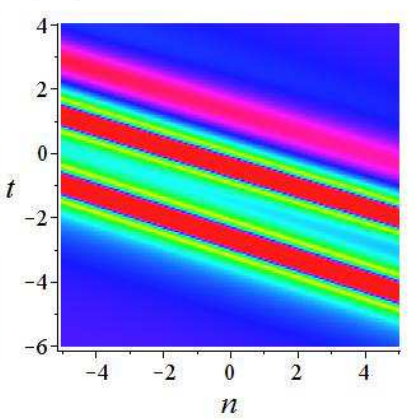

Figure 13: The third-order rational soliton solution Eq. (5.15). (a1)-(a2): Strong interaction, $b_{1}=d_{1}=$ $b_{2}=d_{2}=0$. (b1)-(b2): Weak interaction, $b_{1}=100, d_{1}=b_{2}=d_{2}=0$. (c1)-(c2): Weak interaction, $b_{2}=1000, b_{1}=d_{1}=d_{2}=0$.

solution as follows.

- If $b_{1}=b_{2}=d_{1}=d 2=0$, the third-order rational soliton solutions preserve their shape and speed - cf. Fig. 13 (a1) and (a2).

- If $b_{1}=100$ and $b_{2}=d_{1}=d_{2}=0$, the weak interaction of the third-order rational soliton splits into three parallel solitons - cf. Fig. 13 (b1) and (b2).

- If $b_{2}=10^{3}$ and $b_{1}=d_{1,2}=0$, the weak interaction of the third-order rational soliton splits into three parallelled solitons, which constitute a parallel structure - cf. Fig. 13, (c1)-(c2).

Numerical simulations again illustrate the dynamical behaviour of the third-order rational soliton solution (5.15), which turns out to be similar to the second order solutions cf. Fig. 14(c1). This is probably also due to the energy for the strong interaction being focused around the origin of $(n, t)$, whereas for the weak interaction it is located around three lines.

\section{Conclusions}

An integrable lattice hierarchy associated with Eq. (1.1) has been constructed, and a novel constructive method to determine the generalised perturbation $(n, N-n)$-fold Dar- 
(a1)
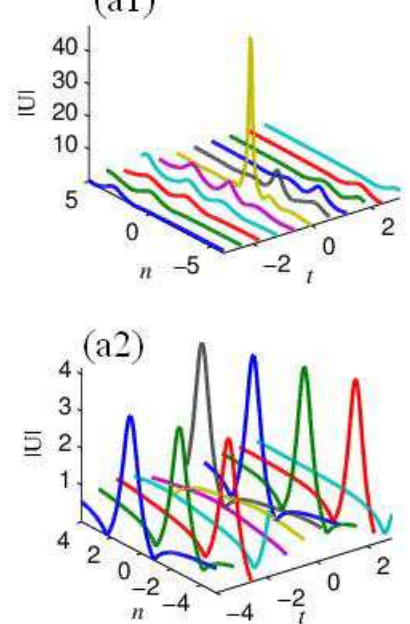

(a3)

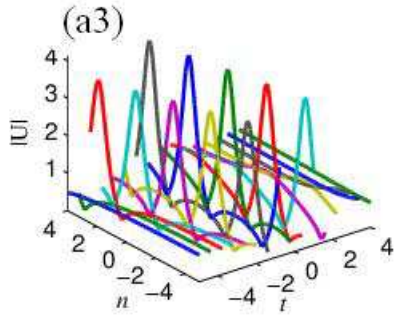

(b1)
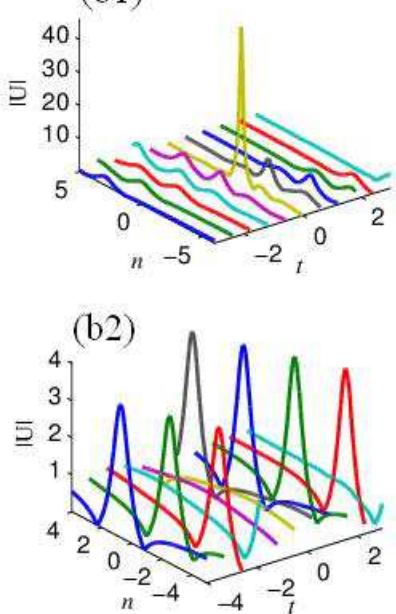

(b3)

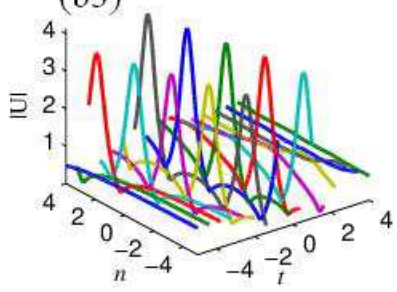

(c1)

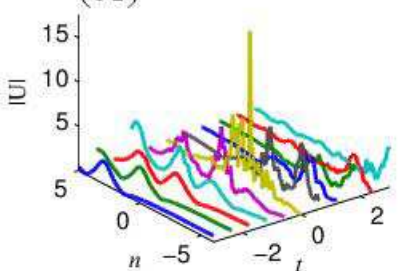

(c2)

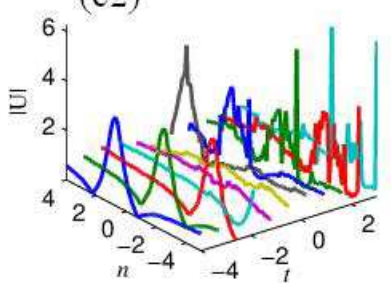

(c3)

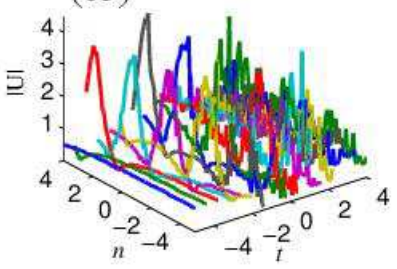

Figure 14: The third-order rational soliton solution(5.15). Left: Exact solution. Middle: Time evolution with no noise. Right: Time evolution with a small $2 \%$ noise.(a1)-(c1): $b_{1}=d_{1}=b_{2}=d_{2}=0$; (a2)-(c2): $b_{1}=100, d_{1}=b_{2}=d_{2}=0 ;(\mathrm{a} 3)-(\mathrm{c} 3): b_{2}=1000, b_{1}=d_{1}=d_{2}=0$.

boux transformation (4.1) and (4.3) of Eq. (1.1) has been presented. This provides a description of higher-order rogue wave and rational soliton solutions in a unified way using specific determinants. The modulational instability of the possible generation mechanism for the rogue wave was considered. The propagation and interaction characteristics of rogue wave and rational soliton solutions and details of their evolution were investigated using numerical simulations for various sets of parameters. A strong interaction yields a weak oscillation and stability, but a weak interaction produces a strong oscillation and instability. These results provide new insight into rogue wave and rational soliton solutions described by Eq. (1.1), relevant to various physical phenomena.

\section{Acknowledgments}

This work has been supported in part by Qin Xin Talents Cultivation Program (QXTCP B201704) of the Beijing Information Science and Technology University, the Beijing Natural Science Foundation Grant No. 1153004, and by NSFC Grants Nos. 11375030, 61471406, 11401031. 


\title{
Appendix A
}

\author{
$\phi^{(1)}=-\frac{1}{1280} C_{1}\left(\frac{5}{2} i\right)^{n} e^{\frac{25}{16} i t} \sqrt{2}\left(675 t^{2}-675 i t^{2}+320 i n-320 n-192 n^{2}+192 i n^{2}-720 n t-720 i n t\right)$, \\ $\psi^{(1)}=\frac{-1+i}{3840} C_{1}\left(\frac{5}{2} i\right)^{n} e^{-i\left(\frac{25}{16} t+\frac{1}{2} \pi n\right)}\left(-2025 t^{2}+1600+1600 i+576 n^{2}-960 n-960 i n+576 i n^{2}-\right.$ \\ $\left.2025 i t^{2}-2160 n t+2160 i n t+3600 t-3600 i t\right)$, \\ $\phi^{(2)}=\frac{1}{819200} C_{1}\left(\frac{5}{2} i\right)^{n} e^{\frac{25}{16} i t}\left(1713600 i t^{2}-122880 i n^{3}+432000 i t^{2} n-131072 i n^{2}-2304000 i t \sqrt{2} d_{1}+\right.$ \\ $1228800 i n \sqrt{2} d_{1}+102400 i n+259200 i t^{2} n^{2}+2304000 i t \sqrt{2} b_{1}-151875 i t^{4}+1228800 i n \sqrt{2} b_{1}-$ \\ $12288 i^{4}-1228800 n \sqrt{2} d_{1}+1228800 n \sqrt{2} b_{1}+967680 n t-324000 n t^{3}-2304000 t \sqrt{2} d_{1}-2304000 t \sqrt{2} b_{1}+$ \\ $\left.460800 t n^{2}+92160 t n^{3}\right)$, \\ $\psi^{(2)}=\frac{1}{4915200} C_{1}\left(\frac{5}{2} i\right)^{n} e^{-i\left(\frac{25}{16} t+\frac{\pi n}{2}\right)}\left(-1620000 i t^{3} \sqrt{2}+5299200 i t \sqrt{2}+27648000 i d_{1} t+36864 i n^{4} \sqrt{2}-\right.$ \\ $7300800 i \sqrt{2} t^{2}+455625 i t^{4} \sqrt{2}+122880 i \sqrt{2} n^{3}-14745600 i n b_{1}-777600 i \sqrt{2} t^{2} n^{2}-36864 \sqrt{2} n^{4}-$ \\ $1296000 \sqrt{2} t^{2} n-2903040 t n \sqrt{2}-276480 \sqrt{2} t n^{3}+972000 \sqrt{2} n t^{3}-184320 n \sqrt{2}+221184 n^{2} \sqrt{2}+$ \\ $1296000 i \sqrt{2} t^{2} n+972000 i \sqrt{2} n t^{3}-455625 t^{4} \sqrt{2}+7300800 \sqrt{2} t^{2}-221184 i n^{2} \sqrt{2}+184320 i n \sqrt{2}-$ \\ $2903040 i t n \sqrt{2}+777600 \sqrt{2} t^{2} n^{2}-122880 \sqrt{2} n^{3}+24576000 i b_{1}-24576000 d_{1}-1620000 \sqrt{2} t^{3}-$ \\ $\left.204800 i \sqrt{2}+14745600 n d_{1}-276480 i \sqrt{2} t n^{3}+204800 \sqrt{2}+27648000 t b_{1}+5299200 t \sqrt{2}\right)$.
}

\section{References}

[1] M.J. Ablowitz and P.A. Clarkson, Solitons, Nonlinear Evolution Equations and Inverse Scattering, Cambridge University Press (1991).

[2] M.J. Ablowitz and J.F. Ladik, Nonlinear differential-difference equations, J. Math. Phys. 16, 598-603 (1975).

[3] M.J. Ablowitz and J.F. Ladik, Nonlinear differential-difference equations and Fourier analysis, J. Math. Phys. 17, 1011-1018 (1976).

[4] M.J. Ablowitz and J.F. Ladik, A nonlinear difference scheme and inverse scattering, Stud. Appl. Math. 55, 213-229 (1976).

[5] M.J. Ablowitz and J.F. Ladik, On the solution of a class of nonlinear partial difference equations, Stud. Appl. Math. 57, 1-12 (1977).

[6] N. Akhmediev and A. Ankiewicz, Modulation instability, Fermi-Pasta-Ulam recurrence, rogue waves, nonlinear phase shift, and exact solutions of the Ablowitz-Ladik equation, Phys. Rev. E 83, 046603 (2011).

[7] A. Ankiewicz, N. Akhmediev and F. Lederer, Approach to first-order exact solutions of the Ablowitz-Ladik equation, Phys. Rev. E 83, 056602 (2011).

[8] A. Ankiewicz, N. Akhmediev and J.M. Soto-Crespo, Discrete rogue waves of the Ablowitz-Ladik and Hirota equations, Phys. Rev. E 82, 026602 (2010).

[9] A. Ankiewicz, N. Devine, M. Ünal, A. Chowdury and N. Akhmediev, Rogue waves and other solutions of single and coupled Ablowitz-Ladik and nonlinear Schrödinger equations, J. Opt. 15, 064008 (2013).

[10] Yu. V. Bludov, V.V. Konotop and N. Akhmediev, Matter rogue waves, Phys. Rev. A 80, 033610 (2009).

[11] Z. Chen, M. Segev and D.N. Christodoulides, Optical spatial solitons: historical overview and recent advances, Rep. Prog. Phys. 75, 086401 (2012). 
[12] E.G. Fan, A family of completely integrable multi-Hamiltonian systems explicitly related to some celebrated equations, J. Math. Phys. 42, 4327-4344 (2001).

[13] B.L. Guo, L.L. Ling and Q.P. Liu, Nonlinear Schrödinger equation: Generalized Darboux transformation and rogue wave solutions, Phys. Rev. E 85, 026607 (2012)

[14] W.X. Ma, A new hierarchy of Liouville integrable generalized Hamiltonian equations and its reduction, Chinese J. Contemp. Math. 13, 79-89 (1992).

[15] W.X. Ma, Darboux transformations for a Lax integrable system in $2 n$ Dimensions, Lett. Math. Phys. 39, 33-49 (1997).

[16] W.X. Ma, A discrete variational identity on semi-direct sums of Lie algebras, J. Phys. A 40, 1505515069 (2007).

[17] W.X. Ma, An application of the Casoratian technique to the 2D Toda lattice equation, Mod. Phys. Lett. B 22, 1815-1825 (2008).

[18] W.X. Ma, Combined Wronskian solutions to the 2D Toda molecule equation, Phys. Lett. A 375, 3931-3935 (2011).

[19] W.X. Ma, Lump solutions to the Kadomtsev-Petviashvili equation, Phys. Lett. A 379, 1975-1978 (2015).

[20] W.X. Ma and B. Fuchssteiner, Algebraic structure of discrete zero curvature equations and master symmetries of discrete evolution equations, J. Math. Phys. 40, 2400-2418 (1999).

[21] W.X. Ma and X.X. Xu, A modified Toda spectral problem and its hierarchy of bi-Hamiltonian lattice, J. Phys. A 37, 1323-1336 (2004).

[22] W.X. Ma and X.X. Xu, Positive and negative hierarchies of integrable lattice models associated with a Hamiltonian pair, Int. J. Theor. Phys. 43, 219-236 (2004).

[23] W.X. Ma, X.X. Xu and Y.F. Zhang, Semidirect sums of Lie algebras and discrete integrable couplings, J. Math. Phys. 47, 053501 (2006).

[24] W.X. Ma, H.Q. Zhang and J.H. Meng, A block matrix loop algebra and bi-integrable couplings of the Dirac equations, E. Asian J. Appl. Math. 3, 171-189 (2013).

[25] T.R. Marchant, Asymptotic solitons on a non-zero mean level, Chaos, Solitons and Fractals 32, 1328-1336 (2007).

[26] Y. Ohta and J.K. Yang, General rogue waves in the focusing and defocusing Ablowitz-Ladik equations, J. Phys. A 47, 255201 (2014).

[27] M. Toda, Theory of Nonlinear Lattices, Springer (1989).

[28] G.Z. Tu, A trace identity and its application to the theory of discrete integrable systems, J. Phys. A 23, 3903-3922 (1990).

[29] M. Wadati, Transformation theories for nonlinear discrete systems, Prog. Theor. Phys. Suppl. 59, 36-63 (1976).

[30] D.S. Wang, Y.R. Shi, W.X. Feng and L. Wen, Dynamical and energetic instabilities of $F=2$ spinor Bose-Einstein condensates in an optical lattice, Physica D 351-352, 30-41 (2017).

[31] D.S. Wang and X. Wei, Integrability and exact solutions of a two-component Korteweg-de Vries system, Appl. Math. Lett. 51, 60-67 (2016).

[32] D.S. Wang, S.J. Yin, Y. Tian and Y. Liu, Integrability and bright soliton solutions to the coupled nonlinear Schrödinger equation with higher-order effects, Appl. Math. Comput. 229, 296-309 (2014).

[33] D.S. Wang, D.J. Zhang and J.K. Yang, Integrable properties of the general coupled nonlinear Schrödinger equations, J. Math. Phys. 51, 023510 (2010).

[34] A.M. Wazwaz, Abundant solitons solutions for several forms of the fifth-order KdV equation by using the tanh method, Appl. Math. Comput. 182, 283-300 (2006).

[35] A.M. Wazwaz, Kadomtsev-Petviashvili hierarchy: $N$-soliton solutions and distinct dispersion relations, Appl. Math. Lett. 52, $74-79$ (2016). 
[36] A.M. Wazwaz, A two-mode modified KdV equation with multiple soliton solutions, Appl. Math. Lett. 70, 1-6 (2017).

[37] X.Y. Wen, Elastic interaction and conservation laws for the nonlinear self-dual network equation in electric circuit, J. Phys. Soc. Jpn. 81, 114006 (2012).

[38] X.Y. Wen and Y.T. Gao, N-Soliton solutions and elastic interaction of the coupled lattice soliton equations for nonlinear waves, Appl. Math. Comput. 219, 99-107 (2012).

[39] X.Y. Wen, Y.T. Gao and L. Wang, Darboux transformation and explicit solutions for the integrable sixth-order KdV equation for nonlinear waves, Appl. Math. Comput. 218, 55-60 (2011).

[40] X.Y. Wen and X.Y. Hu, N-fold Darboux transformation and solitonic interactions for a Volterra lattice system, Adv. Differ. Equ. 2014, 213 (2014).

[41] X.Y. Wen and Z.Y. Yan, Higher-order rational solitons and rogue-like wave solutions of the (2+1)dimensional nonlinear fluid mechanics equations, Commun. Nonlinear Sci. Numer. Simulat. 43, 311-329 (2017).

[42] X.Y. Wen and Z.Y. Yan, Modulational instability and higher-order rogue waves with parameters modulation in a coupled integrable $A B$ system via the generalized Darboux transformation, Chaos, 25, 123115 (2015).

[43] X.Y. Wen, Z.Y. Yan and B.A. Malomed, Higher-order discrete rogue-wave states in the coupled Ablowitz-Ladik equations: Exact solutions and stability, Chaos, 26, 013105 (2016).

[44] X.Y. Wen, Z.Y. Yan and Y.Q. Yang, Dynamics of higher-order rational solitons for the nonlocal nonlinear Schrödinger equation with the self-induced parity-time-symmetric potential, Chaos, 26, 063123 (2016).

[45] X.Y. Wen, Y.Q. Yang and Z.Y. Yan, Generalized perturbation (n, M)-fold Darboux transformations and multi-rogue-wave structures for the modified self-steepening nonlinear Schrödinger equation, Phys. Rev. E 92, 012917 (2015).

[46] T. Xu, H.J. Li, H.J. Zhang, M. Li and S. Lan, Darboux transformation and analytic solutions of the discrete PT-symmetric nonlocal nonlinear Schrödinger equation, Appl. Math. Lett. 63, 88-94 (2017).

[47] Z.Y. Yan, Vector financial rogue waves, Phys. Lett. A 375, 4274 (2011).

[48] Y.F. Zhang and W.X. Ma, A Study on rational solutions to a KP-like equation, Z. Naturforsch. A 70, 263-268 (2015).

[49] Zhaqilao and Z.B. Li, Darboux transformation and multi-solitons for complex mKdV equation, Chin. Phys. Lett. 25, 8-11 (2008).

[50] Zhaqilao, Nth-order rogue wave solutions of the complex modified Korteweg-de Vries equation, Phys. Scr. 87, 065401 (2013). 